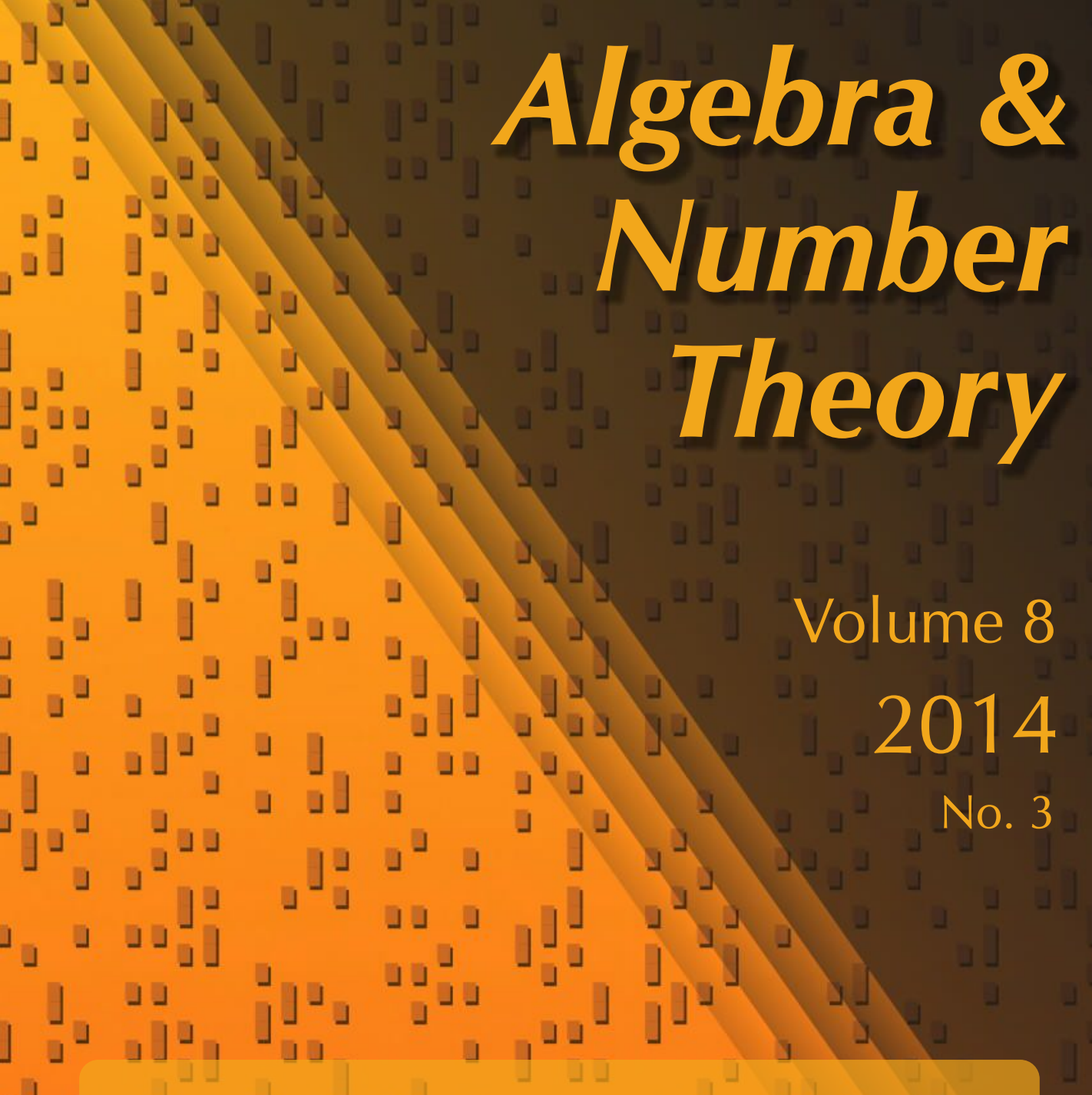

Derived invariants of irregular varieties and Hochschild homology

Luigi Lombardi

\lrcorner

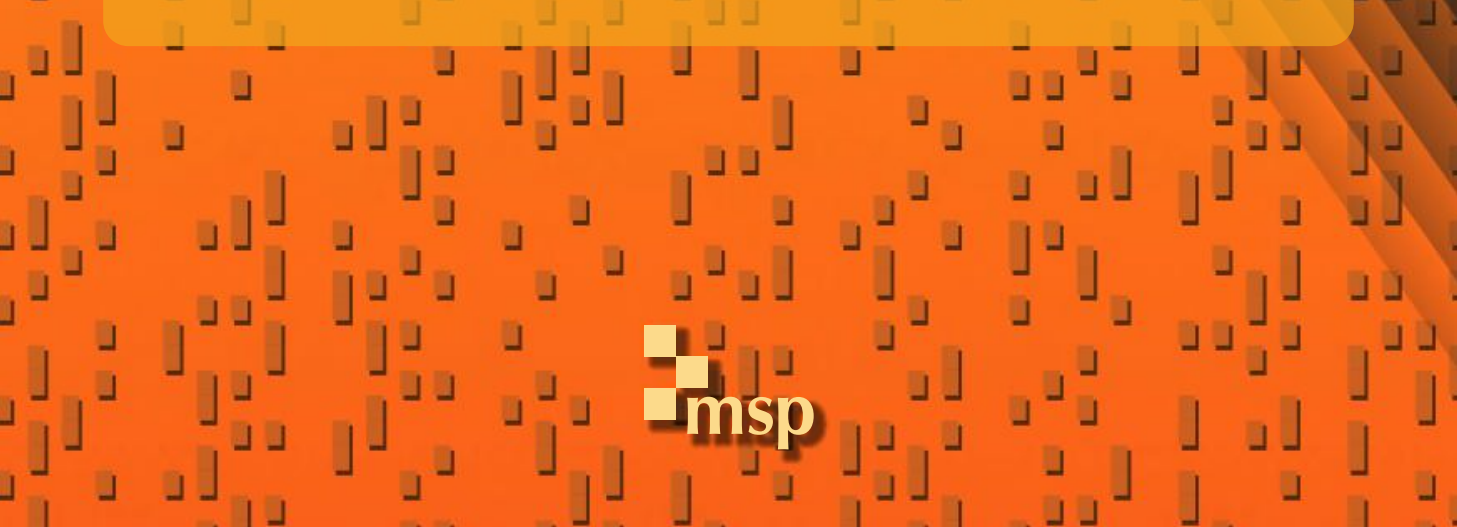




\title{
Derived invariants of irregular varieties and Hochschild homology
}

\author{
Luigi Lombardi
}

\begin{abstract}
We study the behavior of cohomological support loci of the canonical bundle under derived equivalence of smooth projective varieties. This is achieved by investigating the derived invariance of a generalized version of Hochschild homology. Furthermore, using techniques coming from birational geometry, we establish the derived invariance of the Albanese dimension for varieties having nonnegative Kodaira dimension. We apply our machinery to study the derived invariance of the holomorphic Euler characteristic and of certain Hodge numbers for special classes of varieties. Further applications concern the behavior of particular types of fibrations under derived equivalence.
\end{abstract}

\section{Introduction}

It is now well-known that derived equivalent varieties share quite a few invariants. For instance, the dimension, the Kodaira dimension, the numerical dimension and the canonical ring are examples of derived invariants. By describing the behavior under derived equivalence of the Picard variety, Popa and Schnell [2011] establish the derived invariance of the number of linearly independent holomorphic oneforms. In this paper, we study the behavior under derived equivalence of other fundamental objects in the geometry of irregular varieties, i.e., those with positive irregularity $q(X):=h^{0}\left(X, \Omega_{X}^{1}\right)$, such as the cohomological support loci and the Albanese dimension. Applications of our techniques concern the derived invariance of the holomorphic Euler characteristic of varieties with large Albanese dimension and the derived invariance of some of the Hodge numbers of fourfolds again with large Albanese dimension. A further application concerns the behavior of fibrations of derived equivalent threefolds onto irregular varieties. This work is motivated by a well-known conjecture predicting the derived invariance of all Hodge numbers and by a conjecture of Popa (see Conjectures 1.2 and 1.3 and [Popa 2013]).

MSC2010: 14F05.

Keywords: equivalences of derived categories, support loci, Hochschild homology, Hodge numbers,

Picard variety, Rouquier isomorphism. 
The main tool we use to approach the problems described above is the comparison of the cohomology groups of twists by topologically trivial line bundles of the canonical bundles of the varieties in play. This is achieved by studying a generalized version of Hochschild homology that takes into account an important isomorphism due to Rouquier related to derived autoequivalences (see [Rouquier 2011, Théorème 4.18]). In this way, we obtain a theoretical result of independent interest in the study of derived equivalences of smooth projective varieties, which we now present. To begin with, we recall the Hochschild cohomology and homology of a smooth projective variety $X$ :

$$
\begin{aligned}
H H^{*}(X) & :=\bigoplus_{k} \operatorname{Ext}_{X \times X}^{k}\left(i_{*} \mathcal{O}_{X}, i_{*} \widehat{O}_{X}\right), \\
H H_{*}(X) & :=\bigoplus_{k} \operatorname{Ext}_{X \times X}^{k}\left(i_{*} \mathcal{O}_{X}, i_{*} \omega_{X}\right),
\end{aligned}
$$

where $i: X \hookrightarrow X \times X$ is the diagonal embedding of $X$. The space $H H^{*}(X)$ has a structure of ring under composition of morphisms, and $H H_{*}(X)$ is a graded $H H^{*}(X)$-module with the same operation. Results of Căldăraru [2003, Theorem 8.1] and Orlov [2003, Theorem 2.1.8] show that the Hochschild cohomology and homology are derived invariants. More precisely, if $\Phi: \boldsymbol{D}(X) \rightarrow \boldsymbol{D}(Y)$ is an equivalence of derived categories of smooth projective varieties, then it induces an isomorphism of rings $H H^{*}(X) \cong H H^{*}(Y)$ and an isomorphism of graded modules $H H_{*}(X) \cong H H_{*}(Y)$ compatible with the isomorphism $H H^{*}(X) \cong H H^{*}(Y)$. We now present the generalization of Hochschild homology mentioned above. For a triple $(\varphi, L, m) \in \operatorname{Aut}^{0}(X) \times \operatorname{Pic}^{0}(X) \times \mathbb{Z}$, we define the graded $H H^{*}(X)$-module

$$
H H_{*}(X, \varphi, L, m):=\bigoplus_{k} \operatorname{Ext}_{X \times X}^{k}\left(i_{*} \hat{O}_{X},(1, \varphi)_{*}\left(\omega_{X}^{\otimes m} \otimes L\right)\right)
$$

with module structure given by composition of morphisms. We think of these spaces as a "twisted" version of the Hochschild homology of $X$. Lastly, we recall that a derived equivalence $\boldsymbol{D}(X) \cong \boldsymbol{D}(Y)$ induces an isomorphism of algebraic groups, called Rouquier's isomorphism

$$
F: \operatorname{Aut}^{0}(X) \times \operatorname{Pic}^{0}(X) \rightarrow \operatorname{Aut}^{0}(Y) \times \operatorname{Pic}^{0}(Y) .
$$

(An explicit description of $F$ is given in (3) (see [Rouquier 2011, Théorème 4.18; Huybrechts 2006, Proposition 9.45; Rosay 2009, Theorem 3.1]; cf. [Popa and Schnell 2011, footnote, p. 531]).) The following theorem describes the behavior of the twisted Hochschild homology under derived equivalence. Its proof follows the general strategy of the proofs of Orlov and Căldăraru, but further technicalities appear due to the possible presence of nontrivial automorphisms of $X$ and $Y$; see Section 2 for its proof.

Theorem 1.1. Let $\Phi: \boldsymbol{D}(X) \rightarrow \boldsymbol{D}(Y)$ be an equivalence of derived categories of smooth projective varieties defined over an algebraically closed field, and let $m \in \mathbb{Z}$. 
If $F(\varphi, L)=(\psi, M)$ (where $F$ is the Rouquier isomorphism), then $\Phi$ induces an isomorphism of graded modules

$$
H H_{*}(X, \varphi, L, m) \cong H H_{*}(Y, \psi, M, m)
$$

compatible with the isomorphism $H H^{*}(X) \cong H H^{*}(Y)$.

We now move our attention to the main application of Theorem 1.1, namely, the behavior of cohomological support loci under derived equivalence. These loci are defined as

$$
V^{k}\left(\omega_{X}\right):=\left\{L \in \operatorname{Pic}^{0}(X) \mid h^{k}\left(X, \omega_{X} \otimes L\right)>0\right\}
$$

where $X$ is a smooth projective variety and $k \geq 0$ is an integer. From here on, we work over the field of the complex numbers. The $V^{k}\left(\omega_{X}\right)$ 's have been studied for instance in [Green and Lazarsfeld 1987; 1991; Ein and Lazarsfeld 1997; Arapura 1992; Hacon 2004; Pareschi and Popa 2011]. They are one of the most important tools in the birational study of irregular varieties; roughly speaking, they control the geometry of the Albanese map and the fibrations onto lower-dimensional irregular varieties. The following conjecture, and its weaker variant, predicts the behavior of cohomological support loci under derived equivalence. As a matter of notation, we denote by $V^{k}\left(\omega_{X}\right)_{0}$ the union of the irreducible components of $V^{k}\left(\omega_{X}\right)$ passing through the origin.

Conjecture 1.2 [Popa 2013, Conjecture 1.2]. If $X$ and $Y$ are smooth projective derived equivalent varieties, then

$$
V^{k}\left(\omega_{X}\right) \cong V^{k}\left(\omega_{Y}\right) \text { for all } k \geq 0 .
$$

Conjecture 1.3 [Popa 2013, Variant 1.3]. Under the assumptions of Conjecture 1.2, there exist isomorphisms

$$
V^{k}\left(\omega_{X}\right)_{0} \cong V^{k}\left(\omega_{Y}\right)_{0} \quad \text { for all } k \geq 0 .
$$

It is important to emphasize that for all the applications we are interested in (e.g., invariance of the Albanese dimension, invariance of the holomorphic Euler characteristic and invariance of Hodge numbers) it is in fact enough to verify Conjecture 1.3. We also remark that Conjecture 1.2 holds for varieties of general type since the cohomological support loci are birational invariants while derived equivalent varieties of general type are birational by [Kawamata 2002, Theorem 1.4]. Moreover, in [Popa 2013, §2], it has been shown that Conjecture 1.2 holds for surfaces as well.

In Section 3, we try to attack the above conjectures for varieties of arbitrary dimension. To begin with, we show that Theorem 1.1 implies the derived invariance of $V^{0}\left(\omega_{X}\right)$ (see Proposition 3.1). On the other hand, due to the possible presence of nontrivial automorphisms, the study of the derived invariance of the higher 
cohomological support loci is more involved. Nonetheless, by using a version of the Hochschild-Kostant-Rosenberg isomorphism and Brion's structural results on the actions of nonaffine groups on smooth varieties, we are able to show the derived invariance of $V^{1}\left(\omega_{X}\right)_{0}$ (see Corollary 3.4). The next theorem summarizes the main results on the derived invariance of these loci.

Theorem 1.4. Let $X$ and $Y$ be smooth projective derived equivalent varieties. Then the Rouquier isomorphism induces isomorphisms of algebraic sets

(i) $V^{0}\left(\omega_{X}\right) \cong V^{0}\left(\omega_{Y}\right)$,

(ii) $V^{0}\left(\omega_{X}\right) \cap V^{1}\left(\omega_{X}\right) \cong V^{0}\left(\omega_{Y}\right) \cap V^{1}\left(\omega_{Y}\right)$ and

(iii) $V^{1}\left(\omega_{X}\right)_{0} \cong V^{1}\left(\omega_{Y}\right)_{0}$.

We note that (i) also holds if we consider arbitrary powers of the canonical bundle (see Proposition 3.1). We point out also that cases in which the Rouquier isomorphism induces the full isomorphism $V^{1}\left(\omega_{X}\right) \cong V^{1}\left(\omega_{Y}\right)$ occur for instance when either $X$ is of maximal Albanese dimension (see Corollary 5.2) or when the neutral component of the automorphism group, $\operatorname{Aut}^{0}(X)$, is affine (see Remark 3.6); Theorem 1.4 is proved in Section 3.

Next we study Conjectures 1.2 and 1.3 for varieties of dimension three. In the process, we recover Conjecture 1.2 in dimension two as well, making the isomorphisms on cohomological support loci explicit. In the following theorem, we collect all results concerning the behavior of cohomological support loci of derived equivalent threefolds. We denote by $\operatorname{alb}_{X}: X \rightarrow \operatorname{Alb}(X)$ the Albanese map of $X$, and we say that $X$ is of maximal Albanese dimension if $\operatorname{dim} \operatorname{alb}_{X}(X)=\operatorname{dim} X$, i.e., $\mathrm{alb}_{X}$ is generically finite onto its image.

Theorem 1.5. Let $X$ and $Y$ be smooth projective irregular derived equivalent threefolds. Then:

(i) Conjecture 1.3 holds.

(ii) Conjecture 1.2 holds if one of the following hypotheses is satisfied:

(a) $X$ is of maximal Albanese dimension.

(b) $V^{k}\left(\omega_{X}\right)=\operatorname{Pic}^{0}(X)$ for some $k \geq 0$ (for instance, by [Pareschi and Popa 2011 , Theorem $\mathrm{E}], V^{0}\left(\omega_{X}\right)=\operatorname{Pic}^{0}(X)$ whenever $\operatorname{alb}_{X}(X)$ is not fibered in subtori and $\left.V^{0}\left(\omega_{X}\right) \neq \varnothing\right)$.

(c) $\operatorname{Aut}^{0}(X)$ is affine (for instance, by a theorem of Nishi [Matsumura 1963, Theorem 2], this again happens when $\operatorname{alb}_{X}(X)$ is not fibered in subtori).

(iii) If $q(X) \geq 2$, then $\operatorname{dim} V^{k}\left(\omega_{X}\right)=\operatorname{dim} V^{k}\left(\omega_{Y}\right)$ for all $k \geq 0$.

Point (iii) brings evidence to a further variant of Conjecture 1.2 predicting the invariance of the dimensions of cohomological support loci [Popa 2013, Variant 1.4]; partial results for the case $q(X)=1$ are described in Remark 6.10. Since the proofs 
of Theorems 1.4 and 1.5 extend to analogous results regarding cohomological support loci of bundles of holomorphic $p$-forms, when possible, we will prove them in such generality. Please refer to Theorem 4.2 and Section 6 for the proof of Theorem 1.5.

Finally, we move our attention to applications of Theorems 1.4 and 1.5. The first regards the behavior of the Albanese dimension, $\operatorname{dim} \operatorname{alb}_{X}(X)$, under derived equivalence. According to Conjecture 1.3, the Albanese dimension is expected to be preserved under derived equivalence as it can be read off from the dimensions of the $V^{k}\left(\omega_{X}\right)_{0}$ 's (see (5)), which is the case in dimension three thanks to Theorem 1.5. In higher dimension, we establish this invariance for varieties having nonnegative Kodaira dimension $\kappa(X)$ by using the derived invariance of the irregularity and an extension of a result due to Chen, Hacon and Pardini [Hacon and Pardini 2002, Proposition 2.1; Chen and Hacon 2004, Corollary 3.6] on the study of the geometry of the Albanese map via the Iitaka fibration; see Section 5.

Theorem 1.6. Let $X$ and $Y$ be smooth projective derived equivalent varieties. If $\operatorname{dim} X \leq 3$, or if $\operatorname{dim} X>3$ and $\kappa(X) \geq 0$, then

$$
\operatorname{dim} \operatorname{alb}_{X}(X)=\operatorname{dim}_{\operatorname{alb}_{Y}}(Y) .
$$

The second application concerns the holomorphic Euler characteristic. This is expected to be the same for arbitrary derived equivalent smooth projective varieties since the Hodge numbers are expected to be preserved (which is known to hold in dimension up to three [Popa and Schnell 2011, Corollary C]). We deduce this for varieties of large Albanese dimension as a consequence of the previous results and generic vanishing.

Corollary 1.7. Let $X$ and $Y$ be smooth projective derived equivalent varieties. If $\operatorname{dim} \operatorname{alb}_{X}(X)=\operatorname{dim} X$, or if $\operatorname{dim} \operatorname{alb}_{X}(X)=\operatorname{dim} X-1$ and $\kappa(X) \geq 0$, then

$$
\chi\left(\omega_{X}\right)=\chi\left(\omega_{Y}\right) .
$$

An immediate consequence is the derived invariance of two of the Hodge numbers for fourfolds satisfying the hypotheses of Corollary 1.7.

Corollary 1.8. Let $X$ and $Y$ be smooth projective derived equivalent fourfolds. If $\operatorname{dim} \operatorname{alb}_{X}(X)=4$, or if $\operatorname{dim} \operatorname{alb}_{X}(X)=3$ and $\kappa(X) \geq 0$, then

$$
h^{0,2}(X)=h^{0,2}(Y) \quad \text { and } \quad h^{1,3}(X)=h^{1,3}(Y) .
$$

We remark that Popa and Schnell [2011, Corollary 3.4] establish the invariance of $h^{0,2}$ and $h^{1,3}$ under different hypotheses, namely, when $\operatorname{Aut}^{0}(X)$ is not affine (we recall that $h^{0,4}, h^{0,3}, h^{0,1}$ and $h^{1,2}$ are always known to be invariant; see [Popa and Schnell 2011]). Corollaries 1.7 and 1.8 are proved in Section 7. 
We now present our last application in a direction that is one of the main motivations for Conjectures 1.2 and 1.3 as explained in [Popa 2013]. From the classification of Fourier-Mukai equivalences for surfaces [Kawamata 2002; Bridgeland and Maciocia 2001], it is known that, if $X$ admits a fibration $f: X \rightarrow C$ onto a smooth curve of genus $\geq 2$, then any of its Fourier-Mukai partners admits a fibration onto the same curve. Here we use our analysis, and a theorem of Green and Lazarsfeld regarding the properties of positive-dimensional irreducible components of the cohomological support loci, to investigate the behavior of fibrations of derived equivalent threefolds onto irregular varieties. Recall that a smooth variety $X$ is called of Albanese general type if $\mathrm{alb}_{X}$ is nonsurjective and generically finite onto its image. The proof of the next corollary is contained in Proposition 7.3 and Remark 7.4.

Corollary 1.9. Let $X$ and $Y$ be smooth projective derived equivalent threefolds. There exists a morphism $f: X \rightarrow W$ with connected fibers onto a normal variety $W$ of dimension $\leq 2$ such that any smooth model of $W$ is of Albanese general type if and only if $Y$ has a fibration of the same type. Moreover, there exists a morphism $f: X \rightarrow C$ with connected fibers onto a smooth curve $C$ of genus $\geq 2$ if and only if there exists a morphism $h: Y \rightarrow D$ with connected fibers onto a smooth curve $D$ of genus $\geq 2$.

To conclude, we remark that, while the approach in this paper relies in part on techniques of [Popa and Schnell 2011], the key new ingredient is their interaction with the twisted Hochschild homology, introduced and studied here. We are hopeful that this general method will find further applications in the future.

\section{Derived invariance of the twisted Hochschild homology}

In this section, we aim to prove Theorem 1.1. Its proof is based on a technical lemma extending previous computations carried out by Căldăraru [2003, Proposition 8.1] and Orlov [2003, Isomorphism (10)].

Let $X$ and $Y$ be smooth projective varieties defined over an algebraically closed field $K$, and let $p$ and $q$ be the projections from $X \times Y$ onto the first and second factor, respectively. We denote by $\boldsymbol{D}(X):=\boldsymbol{D}^{b}(\mathscr{C} \circ \mathrm{h}(X))$ the bounded derived category of coherent sheaves on a smooth projective variety $X$. When there is no possibility of ambiguity, we use the same symbol to denote a functor and its associated derived functor. An object $\mathscr{E}$ in $\boldsymbol{D}(X \times Y)$ defines Fourier-Mukai functors with kernel $\mathscr{E}$ as

$$
\begin{aligned}
& \Phi_{\mathscr{E}}: \boldsymbol{D}(X) \rightarrow \boldsymbol{D}(Y), \quad \mathscr{F}_{F} \mapsto q_{*}\left(p^{*} \mathscr{F}_{\mathcal{F}} \otimes \mathscr{E}\right), \\
& \Psi_{\mathscr{E}}: \boldsymbol{D}(Y) \rightarrow \boldsymbol{D}(X), \quad \mathscr{G} \mapsto p_{*}\left(q^{*} \mathscr{G} \otimes \mathscr{E}\right) .
\end{aligned}
$$

We say that $X$ and $Y$ are derived equivalent if there exists a $K$-linear exact equivalence of triangulated categories $\Phi: \boldsymbol{D}(X) \rightarrow \boldsymbol{D}(Y)$. By a fundamental result of 
Orlov, any such equivalence is of Fourier-Mukai type; i.e., there exists an object $\mathscr{E}$ in $\boldsymbol{D}(X \times Y)$ such that $\Phi \cong \Phi_{\mathscr{E}}$. Furthermore, the object $\mathscr{E}$ is unique up to isomorphism.

We recall that an equivalence $\Phi_{\mathscr{E}}: \boldsymbol{D}(X) \rightarrow \boldsymbol{D}(Y)$ induces an equivalence

$$
\Phi_{\mathscr{E} *} \nabla_{\mathscr{E}}: \boldsymbol{D}(X \times X) \rightarrow \boldsymbol{D}(Y \times Y)
$$

with kernel

$$
\mathscr{E}^{*} \otimes \mathscr{E}:=p_{13}^{*} \mathscr{C}^{*} \otimes p_{24}^{*} \mathscr{E}
$$

where $\mathscr{E}^{*}:=\boldsymbol{R} \mathscr{H} \operatorname{om}\left(\mathscr{E}, \mathcal{O}_{X \times Y}\right) \otimes p^{*} \omega_{X}[\operatorname{dim} X]$ and $p_{r s}$ is the projection from $X \times X \times Y \times Y$ onto the $(r, s)$-factor [Orlov 2003, Proposition 2.1.7]. Moreover, for any automorphisms $\varphi \in \operatorname{Aut}^{0}(X)$ and $\psi \in \operatorname{Aut}^{0}(Y)$ (here the superscript 0 denotes the neutral component of the corresponding group), we define the embeddings $(1, \varphi): X \hookrightarrow X \times X, x \mapsto(x, \varphi(x))$ and $(1, \psi): Y \hookrightarrow Y \times Y, y \mapsto(y, \psi(y))$. Finally, we denote by $i$ and $j$ the diagonal embeddings of $X$ and $Y$, respectively.

Lemma 2.1. Let $X$ and $Y$ be smooth projective varieties defined over an algebraically closed field, and let $\Phi_{\mathscr{E}}: \boldsymbol{D}(X) \rightarrow \boldsymbol{D}(Y)$ be an equivalence. Denote by $F$ the induced Rouquier isomorphism (see (1)), and let $m \in \mathbb{Z}$. If $F(\varphi, L)=(\psi, M)$, then

$$
\Phi_{\mathscr{E} *} \nabla_{\mathscr{E}}\left((1, \varphi)_{*}\left(\omega_{X}^{\otimes m} \otimes L\right)\right) \cong(1, \psi)_{*}\left(\omega_{Y}^{\otimes m} \otimes M\right) .
$$

Proof. We denote by $t_{r}$ and $t_{r s}$ the projections from $Y \times X \times Y$ onto the $r$-th and $(r, s)$-th factors, respectively. Moreover, we define the morphism $\lambda: Y \times X \times Y \rightarrow$ $X \times X \times Y \times Y$ as $\left(y_{1}, x, y_{2}\right) \mapsto\left(x, \varphi(x), y_{1}, y_{2}\right)$, and we look at the fiber product diagram

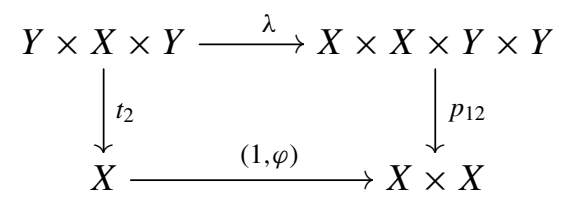

so that, by base change and the projection formula, we get

$$
\begin{aligned}
\Phi_{\mathscr{E} *} \mathbb{E}_{\mathscr{E}}\left((1, \varphi)_{*}\left(\omega_{X}^{\otimes m} \otimes L\right)\right) & \cong p_{34 *}\left(p_{12}^{*}(1, \varphi)_{*}\left(\omega_{X}^{\otimes m} \otimes L\right) \otimes\left(\mathscr{E}^{*} \otimes \mathscr{E}\right)\right) \\
& \cong p_{34 *}\left(\lambda_{*} t_{2}^{*}\left(\omega_{X}^{\otimes m} \otimes L\right) \otimes p_{13}^{*} \mathscr{E}^{*} \otimes p_{24}^{*} \mathscr{E}\right) \\
& \cong p_{34 *} \lambda_{*}\left(t_{2}^{*}\left(\omega_{X}^{\otimes m} \otimes L\right) \otimes \lambda^{*} p_{13}^{*} \mathscr{E}^{*} \otimes \lambda^{*} p_{24}^{*} \mathscr{E}\right) \\
& \cong t_{13 *}\left(t_{2}^{*}\left(\omega_{X}^{\otimes m} \otimes L\right) \otimes t_{21}^{*} \mathscr{E} * t_{23}^{*}(\varphi \times 1)^{* \mathscr{E}}\right) .
\end{aligned}
$$

By [Orlov 2003, p. 535], the equivalence $\Phi_{\mathscr{E}}$ induces an isomorphism $\mathscr{E} \otimes p^{*} \omega_{X} \cong$ $\mathscr{E} \otimes q^{*} \omega_{Y}$. Moreover, by [Popa and Schnell 2011, Lemma 3.1], the condition $F(\varphi, L)=(\psi, M)$ is equivalent to an isomorphism of objects in $\boldsymbol{D}(X \times Y)$

$$
(\varphi \times 1)^{* \mathscr{E}} \otimes p^{*} L \cong(1 \times \psi)_{*} \mathscr{E} \otimes q^{*} M .
$$


Therefore, we get an isomorphism of objects

$$
p^{*}\left(\omega_{X}^{\otimes m} \otimes L\right) \otimes(\varphi \times 1)^{* \mathscr{E}} \cong q^{*}\left(\omega_{Y}^{\otimes m} \otimes M\right) \otimes(1 \times \psi)_{*} \mathscr{E},
$$

and by pulling it back via $t_{23}: Y \times X \times Y \rightarrow X \times Y$, we finally obtain

$$
t_{2}^{*}\left(\omega_{X}^{\otimes m} \otimes L\right) \otimes t_{23}^{*}(\varphi \times 1)^{* \mathscr{E}} \cong t_{3}^{*}\left(\omega_{Y}^{\otimes m} \otimes M\right) \otimes t_{23}^{*}(1 \times \psi)_{*}^{\mathscr{E}} .
$$

At this point, we rewrite the morphism $t_{3}: Y \times X \times Y \rightarrow Y$ as $t_{3}=\sigma_{2} \circ t_{13}$, where $\sigma_{2}: Y \times Y \rightarrow Y$ is the projection onto the second factor. Moreover, we denote by $\rho: Y \times X \rightarrow X \times Y$ the inversion morphism $(y, x) \mapsto(x, y)$. Then by (2) and (4), we obtain

$$
\begin{aligned}
\Phi_{\mathscr{E} * \otimes \mathscr{E}}\left((1, \varphi)_{*}\left(\omega_{X}^{\otimes m} \otimes L\right)\right) & \cong t_{13 *}\left(t_{3}^{*}\left(\omega_{Y}^{\otimes m} \otimes M\right) \otimes t_{21}^{*} \mathscr{E}^{*} \otimes t_{23}^{*}(1 \times \psi)_{*} \mathscr{E}\right) \\
& \cong t_{13 *}\left(t_{13}^{*} \sigma_{2}^{*}\left(\omega_{Y}^{\otimes m} \otimes M\right) \otimes t_{21}^{*} \mathscr{E}^{*} \otimes t_{23}^{*}(1 \times \psi)_{*} \mathscr{E}\right) \\
& \cong \sigma_{2}^{*}\left(\omega_{Y}^{\otimes m} \otimes M\right) \otimes t_{13 *}\left(t_{21}^{*} \mathscr{E}^{*} \otimes t_{23}^{*}(1 \times \psi)_{*} \mathscr{E}\right) \\
& \cong \sigma_{2}^{*}\left(\omega_{Y}^{\otimes m} \otimes M\right) \otimes t_{13 *}\left(t_{12}^{*} \rho^{* \mathscr{E} *} \otimes t_{23}^{*}(1 \times \psi)_{*} \mathscr{E}\right) .
\end{aligned}
$$

Finally, by [Orlov 2003, Proposition 2.1.2], the object $t_{13 *}\left(t_{12}^{*} \rho^{* \mathscr{E} *} \otimes t_{23}^{*}(1 \times \psi)_{*}^{\mathscr{C}}\right)$ in $\boldsymbol{D}(Y \times Y)$ is the kernel of the composition

$$
\Phi_{(1 \times \psi)_{* \mathscr{E}}} \circ \Phi_{\rho^{* \mathscr{E} *}} \cong \psi_{*} \circ \Phi_{\mathscr{E}} \circ \Psi_{\mathscr{E} *} \cong \psi_{*} \circ \operatorname{id}_{\boldsymbol{D}(Y)} \cong \psi_{*},
$$

where we used the fact that $\Psi_{\mathscr{E} *}$ is the right adjoint to $\Phi_{\mathscr{E}}$. On the other hand, since the kernel of the derived functor $\psi_{*}: \boldsymbol{D}(Y) \rightarrow \boldsymbol{D}(Y)$ is the structure sheaf of the graph of $\psi$, i.e., $0_{\Gamma_{\psi}} \cong(1, \psi)_{*} O_{Y}$ [Huybrechts 2006, Example 5.4], we have an isomorphism

$$
t_{13 *}\left(t_{12}^{*} \rho^{* \mathscr{E} *} \otimes t_{23}^{*}(1 \times \psi)_{*} \mathscr{E}\right) \cong(1, \psi)_{*} \mathrm{O}_{Y}
$$

as the kernel of an equivalence is unique up to isomorphism. To recap,

$$
\begin{aligned}
\Phi_{\mathscr{E} *} \nabla_{\mathscr{E}}\left((1, \varphi)_{*}\left(\omega_{X}^{\otimes m} \otimes L\right)\right) & \cong \sigma_{2}^{*}\left(\omega_{Y}^{\otimes m} \otimes M\right) \otimes(1, \psi)_{*} \mathbb{O}_{Y} \\
& \cong(1, \psi)_{*}\left((1, \psi)^{*} \sigma_{2}^{*}\left(\omega_{Y}^{\otimes m} \otimes M\right)\right) \\
& \cong(1, \psi)_{*}\left(\psi^{*}\left(\omega_{Y}^{\otimes m} \otimes M\right)\right) \\
& \cong(1, \psi)_{*}\left(\omega_{Y}^{\otimes m} \otimes M\right) .
\end{aligned}
$$

The last isomorphism follows as the action of $\operatorname{Aut}^{0}(X)$ on $\operatorname{Pic}^{0}(X)$ is trivial [Popa and Schnell 2011, Footnote, p. 531].

Proof of Theorem 1.1. Let $\mathscr{E}$ be the kernel of the equivalence $\Phi$ so that $\Phi \cong \Phi_{\mathscr{E}}$. By Lemma 2.1, the equivalence $\Phi_{\mathscr{E} *} \varpi_{\mathscr{E}}$ induces isomorphisms between the graded 
components of $H H_{*}(X, \varphi, L, m)$ and $H H_{*}(Y, \psi, M, m)$ as follows:

$$
\begin{aligned}
\operatorname{Ext}_{X \times X}^{k}\left(i_{*} O_{X},(1, \varphi)_{*}\left(\omega_{X}^{\otimes m}\right.\right. & \otimes L)) \\
& \cong \operatorname{Ext}_{Y \times Y}^{k}\left(\Phi_{\mathscr{E} * \bigotimes_{\mathscr{E}}}\left(i_{*} \mathcal{O}_{X}\right), \Phi_{\mathscr{E} *} \otimes_{\mathscr{E}}\left((1, \varphi)_{*}\left(\omega_{X}^{\otimes m} \otimes L\right)\right)\right) \\
& \cong \operatorname{Ext}_{Y \times Y}^{k}\left(j_{*} \mathcal{O}_{Y},(1, \psi)_{*}\left(\omega_{Y}^{\otimes m} \otimes M\right)\right) .
\end{aligned}
$$

Moreover, since $\Phi_{\mathscr{E} *} \square_{\mathscr{E}}$ is a functor, it follows that it induces an isomorphism of graded modules.

Theorem 1.1 will be often used in the following weaker form:

Corollary 2.2. Let $X$ and $Y$ be smooth projective derived equivalent varieties defined over an algebraically closed field of characteristic zero. If $F(1, L)=(1, M)$, then for any integers $m$ and $k \geq 0$ there exist isomorphisms

$$
\bigoplus_{q=0}^{k} H^{k-q}\left(X, \Omega_{X}^{\operatorname{dim} X-q} \otimes \omega_{X}^{\otimes m} \otimes L\right) \cong \bigoplus_{q=0}^{k} H^{k-q}\left(Y, \Omega_{Y}^{\operatorname{dim} Y-q} \otimes \omega_{Y}^{\otimes m} \otimes M\right) .
$$

Proof. The corollary is a consequence of Theorem 1.1 and of the general fact that the groups $\operatorname{Ext}_{X \times X}^{k}\left(i_{*} \mathcal{O}_{X}, i_{*} \mathscr{F}\right)$ decompose as $\bigoplus_{q=0}^{k} H^{k-q}\left(X, \Omega_{X}^{\operatorname{dim} X-q} \otimes \omega_{X}^{-1} \otimes \mathscr{F}\right)$ for any coherent sheaf $\mathscr{F}$ and for all $k \geq 0$ [Yekutieli 2003, Corollary 4.7; Swan 1996, Corollary 2.6].

\section{Behavior of cohomological support loci under derived equivalence}

In this section, we study the behavior of cohomological support loci under derived equivalence. Applications of our analysis will be provided in Section 7. From now on, we work over the field of the complex numbers.

3A. Cohomological support loci. Let $X$ be a complex smooth projective irregular variety. Given a coherent sheaf $\mathscr{F}$ on $X$, we define the cohomological support loci of $\mathscr{F}$ as

$$
V_{r}^{k}(\mathscr{F}):=\left\{L \in \operatorname{Pic}^{0}(X) \mid h^{k}(X, \mathscr{F} \otimes L) \geq r\right\}
$$

for all integers $k \geq 0$ and $r \geq 1$. By semicontinuity, these loci are algebraic closed subsets in $\operatorname{Pic}^{0}(X)$. We set $V^{k}(\mathscr{F}):=V_{1}^{k}(\mathscr{F})$, and we denote by $V_{r}^{k}(\mathscr{F})_{0}$ the union of all the irreducible components of $V_{r}^{k}(\mathscr{F})$ passing through the origin of $\operatorname{Pic}^{0}(X)$. By following the work of Pareschi and Popa [2011], we say that $\mathscr{F}$ is a $G V$-sheaf if

$$
\operatorname{codim}_{\operatorname{Pic}^{0}(X)} V^{k}(\mathscr{F}) \geq k \text { for all } k>0 .
$$

In the following, we study the behavior of the loci $V_{r}^{k}(\mathscr{F})$ under equivalence of derived categories where $\mathscr{F}_{F}=\omega_{X}, \omega_{X}^{\otimes m}, \Omega_{X}^{p} \otimes \omega_{X}^{\otimes m}$ with $m, p \in \mathbb{Z}$ and $p \geq 0$. We recall that the cohomological support loci $V^{k}\left(\omega_{X}\right)$ associated to the canonical bundle are invariant under birational modifications for all $k \geq 0$. Furthermore, they 
detect the Albanese dimension of $X$, namely, the dimension of the image of the Albanese map alb $\operatorname{alb}_{X}: X \rightarrow \operatorname{Alb}(X)$, thanks to the following formula [Popa 2013, p. 7] deduced from results of [Green and Lazarsfeld 1987; Lazarsfeld and Popa 2010]:

$$
\operatorname{dim} \operatorname{alb}_{X}(X)=\min _{k=0, \ldots, \operatorname{dim} X}\left\{\operatorname{dim} X-k+\operatorname{codim} V^{k}\left(\omega_{X}\right)_{0}\right\} .
$$

Finally, we point out that, if $\operatorname{dim}_{\operatorname{alb}_{X}}(X)=\operatorname{dim} X-k$, then there are inclusions

$$
V^{k}\left(\omega_{X}\right) \supset V^{k+1}\left(\omega_{X}\right) \supset \cdots \supset V^{\operatorname{dim} X}\left(\omega_{X}\right)=\left\{\mathscr{O}_{X}\right\}
$$

(see [Pareschi and Popa 2011, Proposition 3.14; Green and Lazarsfeld 1987, Theorem 1] or [Ein and Lazarsfeld 1997, Lemma 1.8] for the case $k=0$ ).

3B. Derived invariance of the zeroth cohomological support locus. The following proposition proves and extends Theorem 1.4(i):

Proposition 3.1. Let $X$ and $Y$ be smooth projective varieties, and let $\Phi_{\mathscr{E}}: D(X) \rightarrow$ $\boldsymbol{D}(Y)$ be an equivalence. Denote by $F$ the induced Rouquier isomorphism, and let $m$ and $r$ be integers such that $r \geq 1$. If $L \in V_{r}^{0}\left(\omega_{X}^{\otimes m}\right)$ and $F(1, L)=(\psi, M)$, then $\psi=1$ and $M \in V_{r}^{0}\left(\omega_{Y}^{\otimes m}\right)$. Moreover, $F$ induces an isomorphism of algebraic sets

$$
V_{r}^{0}\left(\omega_{X}^{\otimes m}\right) \cong V_{r}^{0}\left(\omega_{Y}^{\otimes m}\right)
$$

Proof. Let $L$ be a line bundle in $V_{r}^{0}\left(\omega_{X}^{\otimes m}\right)$, and suppose that $F(1, L)=(\psi, M)$ for some $\psi \in \operatorname{Aut}^{0}(Y)$ and $M \in \operatorname{Pic}^{0}(Y)$. By Theorem 1.1 and the adjunction formula, we have

$$
\begin{aligned}
& r \leq h^{0}\left(X, \omega_{X}^{\otimes m} \otimes L\right)=\operatorname{dim} \operatorname{Hom}_{X \times X}\left(i_{*} \mathcal{O}_{X}, i_{*}\left(\omega_{X}^{\otimes m} \otimes L\right)\right) \\
& =\operatorname{dim} \operatorname{Hom}_{Y \times Y}\left(j_{*} \mathfrak{O}_{Y},(1, \psi)_{*}\left(\omega_{Y}^{\otimes m} \otimes M\right)\right) \\
& =\operatorname{dim} \operatorname{Hom}_{Y}\left((1, \psi)^{*} j_{*} O_{Y}, \omega_{Y}^{\otimes m} \otimes M\right) \text {. }
\end{aligned}
$$

Since $(1, \psi)^{*} j_{*} O_{Y}$ is supported on the locus of fixed points of $\psi$ (which is of codimension $\geq 1$ if $\psi \neq 1$ ) and since there are no nonzero morphisms from a torsion sheaf to a locally free sheaf, we must have that $\psi$ is the identity automorphism on $Y$ and consequently that $M \in V_{r}^{0}\left(\omega_{Y}^{\otimes m}\right)$. Therefore, we have an inclusion of algebraic sets $F\left(1, V_{r}^{0}\left(\omega_{X}^{\otimes m}\right)\right) \subset\left(1, V_{r}^{0}\left(\omega_{Y}^{\otimes m}\right)\right)$.

In order to show the reverse inclusion, we consider the right adjoint $\Psi_{\mathscr{E} *}$ to $\Phi_{\mathscr{E}}$ so that $\Psi_{\mathscr{E} *} \circ \Phi_{\mathscr{E}} \cong 1_{\boldsymbol{D}(X)}$ and $\Phi_{\mathscr{E}} \circ \Psi_{\mathscr{E} *} \cong 1_{\boldsymbol{D}(Y)}$. An easy computation shows that, if $F^{\prime}$ is the Rouquier isomorphism induced by $\Psi_{\mathscr{E} *}$, then $F^{\prime}=F^{-1}$ [Lombardi 2013, Lemma 2.1.9]. Hence, by repeating the previous argument, we get an inclusion $F^{-1}\left(1, V_{r}^{0}\left(\omega_{Y}^{\otimes m}\right)\right) \subset\left(1, V_{r}^{0}\left(\omega_{X}^{\otimes m}\right)\right)$ inducing the wanted isomorphism. 
3C. Behavior of higher cohomological support loci under derived equivalence. In this section, we establish the isomorphism $V^{1}\left(\omega_{X}\right)_{0} \cong V^{1}\left(\omega_{Y}\right)_{0}$ of Theorem 1.4. It turns out that, by using the same techniques (i.e., invariance of twisted Hochschild homology and Brion's results on actions of nonaffine groups), one can show a more general result involving cohomological support loci associated to bundles of holomorphic $p$-forms, which we now present.

Theorem 3.2. Let $X$ and $Y$ be smooth projective varieties of dimension d, and let $\Phi_{\mathscr{G}}: \boldsymbol{D}(X) \rightarrow \boldsymbol{D}(Y)$ be an equivalence. Denote by $F$ be the induced Rouquier isomorphism, and let $m$ be an integer. If $L \in \bigcup_{p, q \geq 0} V^{p}\left(\Omega_{X}^{q} \otimes \omega_{X}^{\otimes m}\right)_{0}$ and $F(1, L)=$ $(\psi, M)$, then $\psi=1$ and $M \in \bigcup_{p, q \geq 0} V^{p}\left(\Omega_{Y}^{q} \otimes \omega_{Y}^{\otimes m}\right)_{0}$. Moreover, $F$ induces isomorphisms of algebraic sets

$$
\bigcup_{q=0}^{k} V^{k-q}\left(\Omega_{X}^{d-q} \otimes \omega_{X}^{\otimes m}\right)_{0} \cong \bigcup_{q=0}^{k} V^{k-q}\left(\Omega_{Y}^{d-q} \otimes \omega_{Y}^{\otimes m}\right)_{0} \quad \text { for any } k \geq 0 .
$$

Proof. To begin with, we recall some notation and facts from [Popa and Schnell 2011, Theorem A]. Let $\alpha: \operatorname{Pic}^{0}(Y) \rightarrow \operatorname{Aut}^{0}(X)$ and $\beta: \operatorname{Pic}^{0}(X) \rightarrow \operatorname{Aut}^{0}(Y)$ be morphisms defined as

$$
\alpha(M)=\operatorname{pr}_{1}\left(F^{-1}(1, M)\right) \quad \text { and } \quad \beta(L)=\operatorname{pr}_{1}(F(1, L))
$$

$\left(\operatorname{pr}_{1}\right.$ denotes the projection onto the first factor from the product $\left.\operatorname{Aut}^{0}(\cdot) \times \operatorname{Pic}^{0}(\cdot)\right)$. We denote by $A$ and $B$ the images of $\alpha$ and $\beta$, respectively. We recall that $A$ and $B$ are isogenous abelian varieties.

We first consider the case when $A$ is trivial. Then $F\left(1, \operatorname{Pic}^{0}(X)\right)=\left(1, \operatorname{Pic}^{0}(Y)\right)$, and by Corollary 2.2, we get inclusions

$$
F\left(1, \bigcup_{q} V^{k-q}\left(\Omega_{X}^{d-q} \otimes \omega_{X}^{\otimes m} r\right)\right) \subset\left(1, \bigcup_{q} V^{k-q}\left(\Omega_{Y}^{d-q} \otimes \omega_{Y}^{\otimes m} r\right)\right) \quad \text { for any } k \geq 0 .
$$

In order to prove the reverse inclusions, we note that $B$ is trivial as well and that the Rouquier isomorphism induced by the right adjoint $\Psi_{\mathscr{E} *}$ to $\Phi_{\mathscr{E}}$ is $F^{-1}$. Therefore, a second application of Corollary 2.2 yields inclusions

$F^{-1}\left(1, \bigcup_{q} V^{k-q}\left(\Omega_{Y}^{d-q} \otimes \omega_{Y}^{\otimes m} r\right)\right) \subset\left(1, \bigcup_{q} V^{k-q}\left(\Omega_{X}^{d-q} \otimes \omega_{X}^{\otimes m}\right)\right) \quad$ for any $k \geq 0$, concluding the proof of this case.

We suppose now that both $A$ and $B$ are nontrivial. We first show the following:

Claim 3.3. There are inclusions $F\left(1, \cup_{q} V^{k-q}\left(\Omega_{X}^{d-q} \otimes \omega_{X}^{\otimes m}\right)_{0}\right) \subset\left(1, \operatorname{Pic}^{0}(Y)\right)$ for all integers $m$ and $k \geq 0$.

Proof. Brion's results on actions of nonaffine algebraic groups imply that $X$ is an étale locally trivial fibration $\xi: X \rightarrow A / H$ where $H$ is a finite subgroup of $A$ (the proof of this fact is analogous to the one of [Popa and Schnell 2011, Lemma 2.4]; 
see also [Brion 2010]). Let $Z$ be the smooth and connected fiber of $\xi$ over the origin of $A / H$. Via base change, we get a commutative diagram

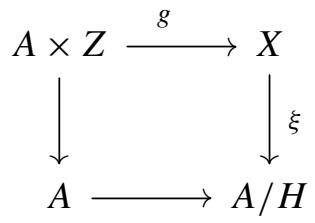

where $g(\varphi, z)=\varphi(z)$. Let $\left(z_{0}, y_{0}\right) \in Z \times Y$ be an arbitrary point, and let

$$
f=\left(f_{1} \times f_{2}\right): A \times B \rightarrow X \times Y
$$

be the orbit map $(\varphi, \psi) \mapsto\left(\varphi\left(z_{0}\right), \psi\left(y_{0}\right)\right)$. In [Popa and Schnell 2011, p. 533], it is shown that

$$
L \in\left(\operatorname{Ker} f_{1}^{*}\right)_{0} \Longrightarrow F(1, L)=(1, M) \text { for some } M \in \operatorname{Pic}^{0}(Y)
$$

(here $\left(\operatorname{Ker} f_{1}^{*}\right)_{0}$ denotes the neutral component of $\left.\operatorname{Ker} f_{1}^{*}\right)$. So it is enough to show the inclusion

$$
\bigcup_{q} V^{k-q}\left(\Omega_{X}^{d-q} \otimes \omega_{X}^{\otimes m}\right)_{0} \subset\left(\operatorname{Ker} f_{1}^{*}\right)_{0} \quad \text { for any } k \geq 0 .
$$

This is achieved by computing cohomology groups on $A \times Z$ via the étale morphism $g$ and by using the fact that these computations are straightforward on $A$. Let $p_{1}$ and $p_{2}$ be the projections from the product $A \times Z$ onto the first and second factors, respectively. By denoting by $v: A \times\left\{z_{0}\right\} \hookrightarrow A \times Z$ the inclusion morphism, we have $g \circ v=f_{1}$. Moreover, via the isomorphism $\operatorname{Pic}^{0}(A \times Z) \cong \operatorname{Pic}^{0}(A) \times \operatorname{Pic}^{0}(Z)$, we obtain $g^{*} L \cong p_{1}^{*} L_{1} \otimes p_{2}^{*} L_{2}$, where $L_{1} \in \operatorname{Pic}^{0}(A)$ and $L_{2} \in \operatorname{Pic}^{0}(Z)$. Note also that $f_{1}^{*} L \cong v^{*} g^{*} L \cong L_{1}$. Finally, for all $L \in \bigcup_{q} V^{k-q}\left(\Omega_{X}^{d-q} \otimes \omega_{X}^{\otimes m}\right)$, there are inclusions

$$
0 \neq \bigoplus_{q=0}^{k} H^{k-q}\left(X, \Omega_{X}^{d-q} \otimes \omega_{X}^{\otimes m} \otimes L\right) \subset \bigoplus_{q=0}^{k} H^{k-q}\left(A \times Z, \Omega_{A \times Z}^{d-q} \otimes \omega_{A \times Z}^{\otimes m} \otimes g^{*} L\right)
$$

[Lazarsfeld 2004, Injectivity Lemma 4.1.14]. Therefore, thanks to Künneth's formula, the sum on the right-hand side of (8) is nonzero only if $f_{1}^{*} L \cong 0_{A}$, i.e., $L \in \operatorname{Ker} f_{1}^{*}$. This shows (7).

By Claim 3.3 and Corollary 2.2, we obtain that for any $k \geq 0$ the Rouquier isomorphism maps

$$
1 \times \bigcup_{q} V^{k-q}\left(\Omega_{X}^{d-q} \otimes \omega_{X}^{\otimes m}\right)_{0} \mapsto 1 \times \bigcup_{q} V^{k-q}\left(\Omega_{Y}^{d-q} \otimes \omega_{Y}^{\otimes m}\right)_{0} .
$$

In complete analogy, one can also show that $M \in \bigcup_{q} V^{k-q}\left(\Omega_{Y}^{d-q} \otimes \omega_{Y}^{\otimes m}\right)_{0} \Rightarrow F^{-1}(1, M)=(1, L) \quad$ for some $L \in \operatorname{Pic}^{0}(X)$. 
This concludes the proof since, by Corollary $2.2, F^{-1}$ maps

$$
1 \times \bigcup_{q} V^{k-q}\left(\Omega_{Y}^{d-q} \otimes \omega_{Y}^{\otimes m}\right)_{0} \mapsto 1 \times \bigcup_{q} V^{k-q}\left(\Omega_{X}^{d-q} \otimes \omega_{X}^{\otimes m}\right)_{0}
$$

for any $k \geq 0$.

The following corollaries yield the proofs of Theorem 1.4(iii) and (ii):

Corollary 3.4. Under the assumptions of Theorem 3.2, the Rouquier isomorphism $F$ induces isomorphisms of algebraic sets

$$
V_{r}^{1}\left(\omega_{X}\right)_{0} \cong V_{r}^{1}\left(\omega_{Y}\right)_{0} \quad \text { for any } r \geq 1 .
$$

Proof. Let $L \in V_{r}^{1}\left(\omega_{X}\right)_{0}$. By Theorem 3.2, we have $F(1, L)=(1, M)$ for some $M \in \operatorname{Pic}^{0}(Y)$, and by Corollary 2.2, we get an isomorphism

$$
H^{1}\left(X, \omega_{X} \otimes L\right) \oplus H^{0}\left(X, \Omega_{X}^{d-1} \otimes L\right) \cong H^{1}\left(Y, \omega_{Y} \otimes M\right) \oplus H^{0}\left(Y, \Omega_{Y}^{d-1} \otimes M\right) .
$$

Moreover, by Serre duality and the Hodge linear-conjugate isomorphism, we obtain equalities

$$
h^{0}\left(X, \Omega_{X}^{d-1} \otimes L\right)=h^{1}\left(X, \omega_{X} \otimes L\right) \quad \text { and } \quad h^{0}\left(Y, \Omega_{Y}^{d-1} \otimes M\right)=h^{1}\left(Y, \omega_{Y} \otimes M\right) .
$$

Hence, $h^{1}\left(X, \omega_{X} \otimes L\right)=h^{1}\left(Y, \omega_{Y} \otimes M\right) \geq r$, and therefore, $F$ induces the wanted isomorphisms as in the proof of Theorem 3.2.

Corollary 3.5. Under the assumptions of Theorem 3.2, and for any integers $l, m, r$ and $s$ with $r, s \geq 1$, the Rouquier isomorphism $F$ induces isomorphisms of algebraic sets

$$
\begin{aligned}
& V_{r}^{0}\left(\omega_{X}^{\otimes m}\right) \cap\left(\bigcup_{q} V^{k-q}\left(\Omega_{X}^{d-q} \otimes \omega_{X}^{\otimes l}\right)\right) \cong V_{r}^{0}\left(\omega_{Y}^{\otimes m}\right) \cap\left(\bigcup_{q} V^{k-q}\left(\Omega_{Y}^{d-q} \otimes \omega_{Y}^{\otimes l}\right)\right), \\
& V_{r}^{0}\left(\omega_{X}^{\otimes m}\right) \cap V_{s}^{1}\left(\omega_{X}\right) \cong V_{r}^{0}\left(\omega_{Y}^{\otimes m}\right) \cap V_{s}^{1}\left(\omega_{Y}\right) .
\end{aligned}
$$

Proof. In Proposition 3.1, we have seen that, if $L \in V_{r}^{0}\left(\omega_{X}^{\otimes m}\right)$, then $F(1, L)=(1, M)$ for some $M \in V_{r}^{0}\left(\omega_{Y}^{\otimes m}\right)$. We argue then as in the proofs of Theorem 3.2 and Corollary 3.4.

Remark 3.6. It is important to note that, whenever $F\left(1, \operatorname{Pic}^{0}(X)\right)=\left(1, \operatorname{Pic}^{0}(Y)\right)$, the proofs of Theorem 3.2 and Corollary 3.4 yield full isomorphisms

$$
\begin{aligned}
\bigcup_{q} V^{k-q}\left(\Omega_{X}^{d-q} \otimes \omega_{X}^{\otimes m}\right) & \cong \bigcup_{q} V^{k-q}\left(\Omega_{Y}^{d-q} \otimes \omega_{Y}^{\otimes m}\right) \quad \text { for any } k \geq 0, \\
V_{r}^{1}\left(\omega_{X}\right) & \cong V_{r}^{1}\left(\omega_{Y}\right) .
\end{aligned}
$$

By Theorem 3.2, this occurs either if $V^{p}\left(\Omega_{X}^{q} \otimes \omega_{X}^{\otimes m}\right)=\operatorname{Pic}^{0}(X)$ for some $p, q \geq 0$ and $m \in \mathbb{Z}$ or if $\operatorname{Aut}^{0}(X)$ is affine (since in this case the abelian variety $A$ in the proof of Theorem 3.2 is trivial). 


\section{Popa's conjectures in dimensions two and three}

In this section, we aim to prove Theorem 1.5(i). In other words, we show that Conjecture 1.3, predicting the derived invariance of cohomological support loci of type $V^{k}\left(\omega_{X}\right)_{0}$, holds in dimension three. The proofs of (ii) and (iii) of the same theorem are postponed to Section 6 since they use the derived invariance of the Albanese dimension, which will be proved in Section 5. Before starting with the proof of Theorem 1.5(i), we make a couple of considerations regarding the case of surfaces.

4A. The case of surfaces. In dimension two, Popa [2013, Theorem 2.1] proves the derived invariance of the full cohomological support loci $V^{k}\left(\omega_{X}\right)$. His proof is based on an explicit computation of cohomological support loci according to the classification of surfaces up to Fourier-Mukai equivalences [Bridgeland and Maciocia 2001]. As an application of Proposition 3.1 and Corollary 3.4, we recover this result by making the isomorphisms between cohomological support loci explicit. More precisely, if $F$ is the Rouquier isomorphism induced by an equivalence of derived categories, then $F\left(1, V_{r}^{k}\left(\omega_{X}\right)\right)=\left(1, V_{r}^{k}\left(\omega_{Y}\right)\right)$ for all integers $k \geq 0$ and $r \geq 1$. Moreover, by using the same techniques, it is possible to show that $F$ induces further isomorphisms $V_{r}^{1}\left(\Omega_{X}^{1}\right) \cong V_{r}^{1}\left(\Omega_{Y}^{1}\right)$ for all $r \geq 1$ (see [Lombardi 2013, Theorem 5.1.2] for a detailed analysis).

Example 4.1 (Elliptic surfaces). Let $X$ be an elliptic surface of Kodaira dimension one and of maximal Albanese dimension (i.e., an isotrivial elliptic surface fibered onto a curve of genus $\geq 2$ ). By following [Beauville 1992], we recall an invariant attached to this type of surfaces. First of all, we note that $X$ admits a unique fibration $f: X \rightarrow C$ onto a curve of genus $\geq 2$ (see for instance [Popa 2013, p. 5]). We then denote by $G$ the general fiber of $f$ and by $\operatorname{Pic}^{0}(X, f)$ the kernel of the pull-back of the inclusion $u: G \hookrightarrow X$

$$
0 \rightarrow \operatorname{Pic}^{0}(X, f) \rightarrow \operatorname{Pic}^{0}(X) \stackrel{u^{*}}{\rightarrow} \operatorname{Pic}^{0}(G)
$$

In [Beauville 1992, (1.6)], it is shown that there exists a finite group $\Gamma^{0}(f)$ and an isomorphism

$$
\operatorname{Pic}^{0}(X, f) \cong f^{*} \operatorname{Pic}^{0}(C) \times \Gamma^{0}(f) .
$$

The group $\Gamma^{0}(f)$ is the invariant mentioned above; it is identified with the group of the connected components of $\operatorname{Pic}^{0}(X, f)$.

We now consider another smooth projective surface $Y$ such that $\boldsymbol{D}(X) \cong \boldsymbol{D}(Y)$. Then, by [Bridgeland and Maciocia 2001, Proposition 4.4], $Y$ is an elliptic surface fibered onto $C$. Moreover, $Y$ is of maximal Albanese dimension as well. To see this, we observe that, since the cohomological support loci are derived invariant in dimension two, we have $\operatorname{dim} \operatorname{alb}_{Y}(Y)=\operatorname{dim} \operatorname{alb}_{X}(X)=2$ thanks to (5). Hence, we 
denote by $g: Y \rightarrow C$ the unique fibration of $Y$ and by $\Gamma^{0}(g)$ its invariant. Pham [2011, Theorem 5.2.7] proves that the invariant $\Gamma^{0}(\cdot)$ attached to this kind of surface is a derived invariant; in other words, he proves that

$$
\Gamma^{0}(f) \cong \Gamma^{0}(g) \text {. }
$$

Here we note that (9) also follows from the derived invariance of the zeroth cohomological support locus. In fact, by results of Popa [2013, p. 5], we know that

$$
V^{0}\left(\omega_{X}\right)=\operatorname{Pic}^{0}(X, f) \cong f^{*} \operatorname{Pic}^{0}(C) \times \Gamma^{0}(f)
$$

and similarly for $V^{0}\left(\omega_{Y}\right)$. Therefore, Proposition 3.1 implies

$$
f^{*} \operatorname{Pic}^{0}(C) \times \Gamma^{0}(f) \cong V^{0}\left(\omega_{X}\right) \cong V^{0}\left(\omega_{Y}\right) \cong g^{*} \operatorname{Pic}^{0}(C) \times \Gamma^{0}(g),
$$

which in particular yields (9).

\section{B. Proof of Theorem 1.5(i).}

Theorem 4.2. Let $X$ and $Y$ be smooth projective threefolds and $\Phi_{\mathscr{E}}: \boldsymbol{D}(X) \rightarrow \boldsymbol{D}(Y)$ an equivalence, and let $F$ be the induced Rouquier isomorphism. Then $F$ induces isomorphisms of algebraic sets

$$
V_{r}^{p}\left(\Omega_{X}^{q}\right)_{0} \cong V_{r}^{p}\left(\Omega_{Y}^{q}\right)_{0} \text { for any } p, q \geq 0 \text { and } r \geq 1 .
$$

Proof. The isomorphisms $V_{r}^{0}\left(\omega_{X}\right) \cong V_{r}^{0}\left(\omega_{Y}\right)$ and $V_{r}^{1}\left(\omega_{X}\right)_{0} \cong V_{r}^{1}\left(\omega_{Y}\right)_{0}$ have been proved in Proposition 3.1 and Corollary 3.4, respectively. On the other hand, the isomorphisms $V_{r}^{3}\left(\omega_{X}\right) \cong V_{r}^{3}\left(\omega_{Y}\right)$ are trivial and follow by Serre duality. We now show the isomorphisms $V_{r}^{2}\left(\omega_{X}\right)_{0} \cong V_{r}^{2}\left(\omega_{Y}\right)_{0}$. To begin with, we note that, by Claim 3.3, if $L \in V_{r}^{2}\left(\omega_{X}\right)_{0}$, then necessarily $F(1, L)=(1, M)$ for some line bundle $M \in \operatorname{Pic}^{0}(Y)$. Moreover, for $k=0,1$, we have equalities $h^{k}\left(X, \omega_{X} \otimes L\right)=h^{k}\left(Y, \omega_{Y} \otimes M\right)$ whenever $L \in V_{r}^{2}\left(\omega_{X}\right)_{0}$ and $F(1, L)=(1, M)$ (see Corollary 2.2). Finally, since the holomorphic Euler characteristic is both a derived invariant in dimension three [Popa and Schnell 2011, Corollary C] and invariant under deformation, we have equalities $\chi\left(\omega_{X} \otimes L\right)=\chi\left(\omega_{X}\right)=\chi\left(\omega_{Y}\right)=\chi\left(\omega_{Y} \otimes M\right)$, from which we easily deduce $h^{2}\left(X, \omega_{X} \otimes L\right)=h^{2}\left(Y, \omega_{Y} \otimes M\right)$. Thus, if $L \in V_{r}^{2}\left(\omega_{X}\right)_{0}$, then $M \in V_{r}^{2}\left(\omega_{Y}\right)_{0}$ and consequently $F$ induces inclusions $F\left(1, V_{r}^{2}\left(\omega_{X}\right)_{0}\right) \subset\left(1, V_{r}^{2}\left(\omega_{Y}\right)_{0}\right)$. Since $F^{-1}$ is the Rouquier isomorphism induced by the right adjoint $\Psi_{\mathscr{E}} *$ to $\Phi_{\mathscr{E}}$, we can repeat the previous argument to obtain the reverse inclusions $F^{-1}\left(1, V_{r}^{2}\left(\omega_{Y}\right)_{0}\right) \subset\left(1, V_{r}^{2}\left(\omega_{X}\right)_{0}\right)$. This in turn yields isomorphisms $V_{r}^{0}\left(\Omega_{X}^{1}\right)_{0} \cong V_{r}^{0}\left(\Omega_{Y}^{1}\right)_{0}$ thanks to Serre duality and the Hodge linear-conjugate isomorphism.

We now prove the isomorphisms $V_{r}^{1}\left(\Omega_{X}^{q}\right)_{0} \cong V_{r}^{1}\left(\Omega_{Y}^{q}\right)_{0}$ for $q=1$, 2. By Claim 3.3, we have $F\left(1, V_{r}^{1}\left(\Omega_{X}^{q}\right)_{0}\right) \subset\left(1, \operatorname{Pic}^{0}(Y)\right)$. By Serre duality and the Hodge linearconjugate isomorphism, $h^{0}\left(X, \Omega_{X}^{1} \otimes L\right)=h^{2}\left(X, \omega_{X} \otimes L\right)$ and $h^{0}\left(Y, \Omega_{Y}^{1} \otimes M\right)=$ $h^{2}\left(Y, \omega_{Y} \otimes M\right)$ for all line bundles $L \in \operatorname{Pic}^{0}(X)$ and $M \in \operatorname{Pic}^{0}(Y)$. Consequently, if 
$L \in V^{0}\left(\Omega_{X}^{1}\right)_{0}$ and $F(1, L)=(1, M)$, then by Corollary 2.2 with $m=0$ and $k=2$ we have $h^{1}\left(X, \Omega_{X}^{2} \otimes L\right)=h^{1}\left(Y, \Omega_{Y}^{2} \otimes M\right)$. At this point, in order to prove the wanted isomorphisms, it is enough to proceed as before. In complete analogy, one can also prove the isomorphisms $V_{r}^{1}\left(\Omega_{X}^{1}\right)_{0} \cong V_{r}^{1}\left(\Omega_{Y}^{1}\right)_{0}$, this time by using Corollary 2.2 with $m=0$ and $k=3$.

\section{Behavior of the Albanese dimension under derived equivalence}

In this section, we prove Theorem 1.6. Our main tool is a generalization of a result due to Chen, Hacon and Pardini saying that, if $f: X \rightarrow Z$ is a nonsingular representative of the Iitaka fibration of a smooth projective variety $X$ of maximal Albanese dimension, then

$$
q(X)-q(Z)=\operatorname{dim} X-\operatorname{dim} Z
$$

[Hacon and Pardini 2002, Proposition 2.1; Chen and Hacon 2004, Corollary 3.6]. We generalize this fact in two ways: (i) we consider all possible values of the Albanese dimension of $X$, and (ii) we replace the litaka fibration with a more general class of morphisms.

Lemma 5.1. Let $X$ and $Z$ be smooth projective varieties and $f: X \rightarrow Z$ a surjective morphism with connected fibers. If the general fiber of $f$ is a smooth variety with surjective Albanese map, then

$$
q(X)-q(Z)=\operatorname{dim}_{\operatorname{alb}}(X)-\operatorname{dim} \operatorname{alb}_{Z}(Z) .
$$

Proof. We follow [Hacon and Pardini 2002, Proposition 2.1; Chen and Hacon 2004, Corollary 3.6]. Due to the functoriality of the Albanese map, we get a commutative diagram

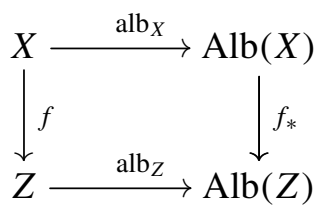

where $f_{*}$ is surjective since $f$ is [Beauville 1996, Remark V.14]. Furthermore, $f_{*}$ has connected fibers. To see this, we denote by $K$ the connected component of $\operatorname{Ker} f_{*}$ through the origin and set $A:=\operatorname{Alb}(X) / K$. Then the natural map $v: A \rightarrow \operatorname{Alb}(Y)$ is étale and $f$ factors through the induced map $Y \times \operatorname{Alb}(Y) A \rightarrow Y$, which is étale of the same degree as $v$. Since $f$ has connected fibers, we see that $v$ is an isomorphism and $K=\operatorname{Ker} f_{*}$.

We now show that the image of a general fiber $P$ of $f$ via $\mathrm{alb}_{X}$ is a translate of Ker $f_{*}$. Since $\mathrm{alb}_{P}$ is surjective, the image of $P$ via $\mathrm{alb}_{X}$ is a translate of a subtorus of Ker $f_{*}$. Furthermore, since $P$ moves in a continuous family, such images are all translates of a fixed subtorus $T \subset \operatorname{Ker} f_{*}$. Our next step is to show $T=\operatorname{Ker} f_{*}$. By 
setting $B:=\operatorname{Alb}(X) / T$, we see that the induced morphism $X \rightarrow B$ maps a general fiber of $f$ to a point. Therefore, it induces a rational map $h: Z \rightarrow B$, which is a morphism since $B$ is an abelian variety. Furthermore, $h(Z)$ generates the abelian variety $B$ since the image of the Albanese map generates the Albanese variety. This leads to the inequality

$$
\operatorname{dim} B \leq q(Z)=q(X)-\operatorname{dim} \operatorname{Ker} f_{*},
$$

which in turn yields $\operatorname{dim} T \geq \operatorname{dim} \operatorname{Ker} f_{*}$ as $\operatorname{dim} B=q(X)-\operatorname{dim} T$. For dimension reasons, we get then $T=\operatorname{Ker} f_{*}$. In particular, this says that $\operatorname{alb}_{X}(X)$ is fibered in tori of dimension $q(X)-q(Z)$ over $\operatorname{alb}_{Z}(Z)$, and by the theorem on the dimension of the fibers of a morphism, we get the stated equality.

Proof of Theorem 1.6. We begin with the case $\operatorname{dim} X \leq 3$. In Sections 4A and $4 \mathrm{~B}$, we have seen that in dimension up to three the cohomological support loci associated to the canonical bundle around the origin are derived invariant, i.e., $V^{k}\left(\omega_{X}\right)_{0} \cong V^{k}\left(\omega_{Y}\right)_{0}$ for all $k \geq 0$. Therefore, (5), in combination with the fact that derived equivalent varieties have the same dimension, immediately leads to $\operatorname{dim} \operatorname{alb}_{X}(X)=\operatorname{dim}_{\operatorname{alb}_{Y}}(Y)$.

We now assume $\operatorname{dim} X>3$ and $\kappa(X) \geq 0$. If $\kappa(X)=\kappa(Y)=0$, then the Albanese maps of $X$ and $Y$ are surjective by [Kawamata 1981, Theorem 1]. Thus, the Albanese dimensions of $X$ and $Y$ are $q(X)$ and $q(Y)$, respectively, which are equal by work of Popa and Schnell [2011, Corollary B].

We now suppose $\kappa(X)=\kappa(Y)>0$. Since the problem is invariant under birational modification, with a little abuse of notation, we consider nonsingular representatives $f: X \rightarrow Z$ and $g: Y \rightarrow W$ of the Iitaka fibrations of $X$ and $Y$, respectively [Mori 1987, (1.10)]. As the canonical rings of $X$ and $Y$ are isomorphic [Orlov 2003, Corollary 2.1.9], it turns out that $Z$ and $W$ are birational varieties (see [Mori 1987, Proposition 1.4] or [Toda 2006, p. 13]). By [Kawamata 1981, Theorem 1], the morphisms $f$ and $g$ satisfy the hypotheses of Lemma 5.1, which yields

$q(X)-\operatorname{dim} \operatorname{alb}_{X}(X)=q(Z)-\operatorname{dim}_{\operatorname{alb}}(Z)$

$$
=q(W)-\operatorname{dim} \operatorname{alb}_{W}(W)=q(Y)-\operatorname{dim} \operatorname{alb}_{Y}(Y) .
$$

We conclude as $q(X)=q(Y)$.

As an application of Theorem 1.6, we have the following:

Corollary 5.2. Let $X$ and $Y$ be smooth projective derived equivalent varieties with $X$ of maximal Albanese dimension. If $F$ denotes the induced Rouquier isomorphism and $F(1, L)=(\psi, M)$ with $L \in V_{r}^{1}\left(\omega_{X}\right)$, then $\psi=1$ and $M \in V_{r}^{1}\left(\omega_{Y}\right)$. Moreover, $F$ induces isomorphisms of algebraic sets

$$
V_{r}^{1}\left(\omega_{X}\right) \cong V_{r}^{1}\left(\omega_{Y}\right) \quad \text { for any } r \geq 1 .
$$


Proof. We have $\kappa(X) \geq 0$ since $X$ is of maximal Albanese dimension. Hence, Theorem 1.6 ensures that $Y$ is of maximal Albanese dimension as well. We apply then Corollary 3.5 after having noted the inclusions $V_{r}^{1}\left(\omega_{X}\right) \subset V^{0}\left(\omega_{X}\right)$ and $V_{r}^{1}\left(\omega_{Y}\right) \subset V^{0}\left(\omega_{Y}\right)($ see $(6))$.

\section{End of the proof of Theorem $\mathbf{1 . 5}$}

6A. Proof of Theorem 1.5(ii). The following two propositions prove and extend Theorem 1.5(ii):

Proposition 6.1. Let $X$ and $Y$ be smooth projective derived equivalent threefolds, and let $F$ be the induced Rouquier isomorphism. Assume that either $\operatorname{Aut}^{0}(X)$ is affine or that $V^{p}\left(\Omega_{X}^{q} \otimes \omega_{X}^{\otimes m}\right)=\operatorname{Pic}^{0}(X)$ for some $m, p, q \in \mathbb{Z}$ with $p, q \geq 0$. Then $F$ induces isomorphisms of algebraic sets

$$
V_{r}^{p}\left(\Omega_{X}^{q}\right) \cong V_{r}^{p}\left(\Omega_{Y}^{q}\right) \text { for all } p, q \geq 0 \text { and } r \geq 1 .
$$

Proof. By Remark 3.6, we have $F\left(1, \operatorname{Pic}^{0}(X)\right)=\left(1, \operatorname{Pic}^{0}(Y)\right)$. The isomorphisms $V_{r}^{0}\left(\omega_{X}\right) \cong V_{r}^{0}\left(\omega_{Y}\right)$ and $V_{r}^{1}\left(\omega_{X}\right) \cong V_{r}^{1}\left(\omega_{Y}\right)$ hold by Proposition 3.1 and Remark 3.6, respectively. The isomorphisms $V_{r}^{2}\left(\omega_{X}\right) \cong V_{r}^{2}\left(\omega_{Y}\right)$ follow since in dimension three $\chi\left(\omega_{X}\right)=\chi\left(\omega_{Y}\right)$ [Popa and Schnell 2011, Corollary C].

We now establish the isomorphisms $V_{r}^{1}\left(\Omega_{X}^{2}\right) \cong V_{r}^{1}\left(\Omega_{Y}^{2}\right)$. Let $L \in V_{r}^{1}\left(\Omega_{X}^{2}\right)$ so that $F(1, L)=(1, M)$ for some $M \in \operatorname{Pic}^{0}(Y)$. By Corollary 2.2 with $m=0$ and $k=2$, Serre duality and the Hodge linear-conjugate isomorphism, we get $h^{1}\left(X, \Omega_{X}^{2} \otimes L\right)=h^{1}\left(Y, \Omega_{Y}^{2} \otimes M\right)$. This shows that $F$ maps $1 \times V_{r}^{1}\left(\Omega_{X}^{2}\right) \mapsto$ $1 \times V_{r}^{1}\left(\Omega_{Y}^{2}\right)$, inducing the wanted isomorphisms as in Proposition 3.1. Finally, the isomorphisms $V_{r}^{1}\left(\Omega_{X}^{1}\right) \cong V_{r}^{1}\left(\Omega_{Y}^{1}\right)$ are deduced in the same way by using Corollary 2.2 with $m=0$ and $k=3$.

Proposition 6.2. Let $X$ and $Y$ be smooth projective derived equivalent threefolds, and let $F$ be the induced Rouquier isomorphism. If $X$ is of maximal Albanese dimension, then $F$ induces isomorphisms of algebraic sets

$$
V_{r}^{k}\left(\omega_{X}\right) \cong V_{r}^{k}\left(\omega_{Y}\right) \quad \text { for all } k \geq 0 \text { and } r \geq 1 .
$$

Proof. Proposition 3.1 and Corollary 5.2 yield the isomorphisms $V_{r}^{k}\left(\omega_{X}\right) \cong V_{r}^{k}\left(\omega_{Y}\right)$ for any $k \neq 2$, so we only focus on the remaining case. Since $X$ is of maximal Albanese dimension, we obtain an inclusion $V_{r}^{2}\left(\omega_{X}\right) \subset V^{0}\left(\omega_{X}\right)$ (see (6)) leading to a further inclusion $F\left(1, V_{r}^{2}\left(\omega_{X}\right)\right) \subset\left(1, \operatorname{Pic}^{0}(Y)\right)$ thanks to Proposition 3.1. Hence, by Corollary 2.2, $h^{k}\left(X, \omega_{X} \otimes L\right)=h^{k}\left(Y, \omega_{Y} \otimes M\right)$ whenever $F(1, L)=(1, M)$ with $L \in V_{r}^{2}\left(\omega_{X}\right)$ and $k=0,1$. Moreover, we get $h^{2}\left(X, \omega_{X} \otimes L\right)=h^{2}\left(Y, \omega_{Y} \otimes M\right)$ since $\chi\left(\omega_{X}\right)=\chi\left(\omega_{Y}\right)$ [Popa and Schnell 2011, Corollary C]. Therefore, $F$ maps $1 \times V_{r}^{2}\left(\omega_{X}\right) \mapsto 1 \times V_{r}^{2}\left(\omega_{Y}\right)$, and by arguing as in Proposition 3.1, $F^{-1}$ maps $1 \times V_{r}^{2}\left(\omega_{Y}\right) \mapsto 1 \times V_{r}^{2}\left(\omega_{X}\right)$, finishing the proof. 
6B. Proof of Theorem 1.5(iii). We show now the proof of Theorem 1.5(iii). Before jumping into technicalities, we first present the plan of its proof.

Thanks to Propositions 6.1 and 6.2, we can assume that $X$ is a threefold with $\operatorname{dim} \operatorname{alb}_{X}(X) \leq 2$ and $V^{0}\left(\omega_{X}\right) \subsetneq \operatorname{Pic}^{0}(X)$ and with nonaffine automorphism group $\operatorname{Aut}^{0}(X)$. In particular, we can suppose that $X$ is not of general type and that $\chi\left(\omega_{X}\right)=0$ [Popa and Schnell 2011, Corollary 2.6]. Thanks to Proposition 3.1, Theorem 1.6 and [Popa and Schnell 2011, Theorem A(1)], the Fourier-Mukai partner $Y$ of $X$ satisfies the same hypotheses as $X$. Hence, Theorem 1.5(iii) follows as soon as we classify $\operatorname{dim} V^{i}\left(\omega_{X}\right)$ in terms of derived invariants. This classification is carried out in the following Propositions 6.5-6.9 where $\operatorname{dim} V^{1}\left(\omega_{X}\right)$ and $\operatorname{dim} V^{2}\left(\omega_{X}\right)$ are computed in terms of $\kappa(X), q(X), \operatorname{dim}_{\operatorname{alb}}(X)$ and $\operatorname{dim} V^{0}\left(\omega_{X}\right)$.

The main tools we use towards the proofs of Propositions 6.5-6.9 are generic vanishing theorems [Green and Lazarsfeld 1987, Theorem 1; Pareschi and Popa 2011, Theorem 5.8], Kollár's result on higher direct images of the canonical bundle [Kollár 1986b, Theorem 3.1; 1986a, Theorem 2.1 and Proposition 7.6] and the classification of smooth projective surfaces (see for instance [Beauville 1996]). The following two lemmas will be useful to our analysis:

Lemma 6.3. Let $X$ and $Y$ be smooth projective varieties and $f: X \rightarrow Y$ be a surjective morphism with connected fibers. If h denotes the dimension of the general fiber of $f$, then

$$
f^{*} V^{k}\left(\omega_{Y}\right) \subset V^{k+h}\left(\omega_{X}\right) \text { for any } k=0, \ldots, \operatorname{dim} Y .
$$

Proof. By [Kollár 1986a, Theorem 2.1 and Proposition 7.6], we have $R^{h} f_{*} \omega_{X} \cong \omega_{Y}$ and $R^{k} f_{*} \omega_{X}=0$ for $k>h$. Moreover, by [Kollár 1986b, Theorem 3.1], we obtain decompositions

$$
H^{k+h}\left(X, \omega_{X} \otimes f^{*} L\right) \cong H^{k}\left(Y, \omega_{Y} \otimes L\right) \oplus \bigoplus_{l \neq k} H^{l}\left(Y, R^{h+k-l} f_{*} \omega_{X} \otimes L\right)
$$

for any $L \in \operatorname{Pic}^{0}(Y)$. At this point, it is enough to note that the pull-back homomorphism $f^{*}: \operatorname{Pic}^{0}(Y) \rightarrow \operatorname{Pic}^{0}(X)$ is injective as the fibers of $f$ are connected.

Lemma 6.4. Let $X$ be a smooth projective variety with $\kappa(X)=-\infty$. Then $V^{0}\left(\omega_{X}^{\otimes m}\right)=\varnothing$ for any $m>0$.

Proof. Suppose that $L \in V^{0}\left(\omega_{X}^{\otimes m}\right)$ for some $m>0$. By [Chen and Hacon 2004, Theorem 3.2], we can assume that $L$ is a line bundle of finite order, say, of order $e$. If $\mathrm{O}_{X} \rightarrow \omega_{X}^{\otimes m} \otimes L$ is a nonzero section of $\omega_{X}^{\otimes m} \otimes L$, then it induces a nonzero section $\mathfrak{O}_{X} \rightarrow \omega_{X}^{\otimes m e}$; this yields a contradiction as $\kappa\left(\omega_{X}\right)=-\infty$.

Proposition 6.5. Let $X$ be a smooth projective threefold such that $\kappa(X)=2$, $\operatorname{dim} \operatorname{alb}_{X}(X)=2, \chi\left(\omega_{X}\right)=0$ and $V^{0}\left(\omega_{X}\right) \varsubsetneqq \operatorname{Pic}^{0}(X)$. If $q(X)=2$, then we have (i) $\operatorname{dim} V^{2}\left(\omega_{X}\right)=0$, (ii) $\operatorname{dim} V^{1}\left(\omega_{X}\right)=1$ if and only if $\operatorname{dim} V^{0}\left(\omega_{X}\right)=1$ and 
(iii) $\operatorname{dim} V^{1}\left(\omega_{X}\right)=0$ if and only if $\operatorname{dim} V^{0}\left(\omega_{X}\right) \leq 0$. If $q(X)>2$, then we have $\operatorname{dim} V^{1}\left(\omega_{X}\right)=\operatorname{dim} V^{2}\left(\omega_{X}\right)=q(X)-1$.

Proof. Since the problem is invariant under birational modification, with a little abuse of notation, we consider a nonsingular representative $f: X \rightarrow S$ of the litaka fibration of $X$ [Mori 1987, (1.10)] so that $X$ and $S$ are smooth varieties and $f$ is an algebraic fiber space. We divide the proof into three cases according to the values of the Albanese dimension of $S$.

Case I: $\operatorname{dim} \operatorname{alb}_{S}(S)=2$. By the classification theory of smooth projective surfaces, $S$ is either a surface of general type, or birational to an abelian surface, or birational to an elliptic surface fibered onto a curve of genus $\geq 2$. Moreover, by Lemma 5.1, we have $q(X)=q(S)$.

If $S$ is of general type, then by Castelnuovo's theorem [Beauville 1996, Theorem $X .4]$ we have $\chi\left(\omega_{S}\right)>0$ and hence $V^{0}\left(\omega_{S}\right)=\operatorname{Pic}^{0}(S)$. Therefore, by Lemma 6.3, we get $V^{1}\left(\omega_{X}\right)=\operatorname{Pic}^{0}(X)$, and consequently, $V^{0}\left(\omega_{X}\right)=\operatorname{Pic}^{0}(X)$ since $\chi\left(\omega_{X}\right)=0$ and $V^{2}\left(\omega_{X}\right) \subsetneq \mathrm{Pic}^{0}(X)$ (see (5)). This contradicts our hypotheses, and hence, this case does not occur.

If $S$ is birational to an abelian surface, then we have $q(X)=q(S)=2$ and $f^{*} \operatorname{Pic}^{0}(S)=\operatorname{Pic}^{0}(X)$. By using [Kollár 1986b, Theorem 3.1], we obtain decompositions

$$
H^{2}\left(X, \omega_{X} \otimes f^{*} L\right) \cong H^{2}\left(S, f_{*} \omega_{X} \otimes L\right) \oplus H^{1}\left(S, R^{1} f_{*} \omega_{X} \otimes L\right)
$$

for any $L \in \operatorname{Pic}^{0}(S)$. Moreover, we note that $R^{1} f_{*} \omega_{X} \cong \omega_{S}$ and $R^{2} f_{*} \omega_{X}=0$ [Kollár 1986a, Proposition 7.6 and Theorem 2.1]. Therefore, since by [Pareschi and Popa 2011, Theorem 5.8] $f_{*} \omega_{X}$ is a GV-sheaf on $S$ (i.e., $\operatorname{codim}_{\operatorname{Pic}^{0}(S)} V^{k}\left(f_{*} \omega_{X}\right) \geq k$ for $k>0$ ), we get $\operatorname{dim} V^{2}\left(\omega_{X}\right)=0$. At this point, the statements (ii) and (iii) of the proposition follow as $\chi\left(\omega_{X}\right)=0$ and $\operatorname{dim} V^{1}\left(\omega_{X}\right) \geq 0$ (note that $O_{X} \in V^{1}\left(\omega_{X}\right)$ since $q(X)=2)$.

If $S$ is birational to an elliptic surface $h: S \rightarrow C$ fibered onto a curve $C$ of genus $g(C)=q(S)-1=q(X)-1 \geq 2$, then $X$ is fibered onto $C$ as well. Therefore, we have $V^{0}\left(\omega_{C}\right)=\operatorname{Pic}^{0}(C)$, and consequently, $V^{2}\left(\omega_{X}\right)$ is of codimension one in $\operatorname{Pic}^{0}(X)$ by Lemma 6.3 and (5). Since $\chi\left(\omega_{X}\right)=0, V^{1}\left(\omega_{X}\right)$ is of codimension one as well.

Case II: $\operatorname{dim}_{\operatorname{alb}_{S}}(S)=1$. We have $q(X)=q(S)+1$ by Lemma 5.1. Moreover, $\mathrm{alb}_{S}$ has connected fibers, and by [Beauville 1996, Proposition V.15], $\operatorname{alb}_{S}(S)$ is a smooth curve of genus $q(S)$. We distinguish two subcases: $q(S)=1$ and $q(S) \geq 2$.

If $q(S)=1$, then $q(X)=2$ and $\operatorname{alb}_{X}$ is surjective. Let $X \stackrel{b}{\rightarrow} Z \rightarrow \operatorname{Alb}(X)$ be the Stein factorization of $\operatorname{alb}_{X}$, and let $b^{\prime}: X^{\prime} \rightarrow Z^{\prime}$ be a nonsingular representative of $b$. We note that $Z^{\prime}$ is a smooth surface with $q\left(Z^{\prime}\right)=2$ and of maximal Albanese dimension. Therefore, either $Z^{\prime}$ is of general type or it is birational to an abelian surface. However, we have just seen that $Z^{\prime}$ cannot possibly be of general type; 
therefore, $Z^{\prime}$ is birational to an abelian surface, and the same calculations of the previous case apply.

If $q(S) \geq 2$, then the Albanese map of $S$ induces a fibration of $S$ onto a smooth curve $C$ of genus $g(C)=q(S)$. Therefore, $X$ is fibered onto $C$ as well, and we conclude as in the previous case.

Case III: $\operatorname{dim} \operatorname{alb}_{S}(S)=0$. As we have seen in the proof of Lemma 5.1, the image of a general fiber of $f$ is mapped via $\mathrm{alb}_{X}$ onto a fiber of the induced morphism $f_{*}: \operatorname{Alb}(X) \rightarrow \operatorname{Alb}(S)$. On the other hand, if $\operatorname{dim}_{\operatorname{alb}}(S)=0$, then $\operatorname{Alb}(S)$ is trivial. This yields a contradiction, and therefore, this case does not occur.

Proposition 6.6. Let $X$ be a smooth projective threefold such that $\kappa(X)=2$, $\operatorname{dim} \operatorname{alb}_{X}(X)=1, \chi\left(\omega_{X}\right)=0$ and $V^{0}\left(\omega_{X}\right) \subsetneq \operatorname{Pic}^{0}(X)$. If $q(X)=1$, then we have $\operatorname{dim} V^{1}\left(\omega_{X}\right) \leq 0$ and $\operatorname{dim} V^{2}\left(\omega_{X}\right)=0$. On the other hand, if $q(X)>1$, then we have $V^{1}\left(\omega_{X}\right)=V^{2}\left(\omega_{X}\right)=\operatorname{Pic}^{0}(X)$.

Proof. As in the previous proof, we denote by $f: X \rightarrow S$ a nonsingular representative of the Iitaka fibration of $X$. We distinguish two cases: $\operatorname{dim}_{\operatorname{alb}}(S)=0$ and $\operatorname{dim} \operatorname{alb}_{S}(S)=1$.

If $\operatorname{dim} \operatorname{alb}_{S}(S)=0$, then we have $q(S)=0$ and therefore $q(X)=1$ by Lemma 5.1. Moreover, by [Ueno 1973, Lemma 2.11], $\mathrm{alb}_{X}$ is surjective and has connected fibers. We set $E:=\operatorname{Alb}(X)$ and $a:=\operatorname{alb}_{X}$ and note that by [Kollár 1986a, Proposition 7.6] there is an isomorphism $R^{2} a_{*} \omega_{X} \cong \mathcal{O}_{E}$. Finally, by [Kollár 1986b, Theorem 3.1], we get isomorphisms

$$
H^{2}\left(X, \omega_{X} \otimes a^{*} L\right) \cong H^{1}\left(E, R^{1} a_{*} \omega_{X} \otimes L\right) \oplus H^{0}(E, L)
$$

for any $L \in \operatorname{Pic}^{0}(E) \cong \operatorname{Pic}^{0}(X)$. By [Hacon 2004, Corollary 4.2], $R^{1} a_{*} \omega_{X}$ is a $\mathrm{GV}$-sheaf on $E$. Hence, $\operatorname{dim} V^{2}\left(\omega_{X}\right)=0$, and consequently, $V^{1}\left(\omega_{X}\right)$ is either empty or zero-dimensional as $V^{0}\left(\omega_{X}\right) \subsetneq \operatorname{Pic}^{0}(X)$ and $\chi\left(\omega_{X}\right)=0$.

We now suppose $\operatorname{dim} \operatorname{alb}_{S}(S)=1$. In this case, $\operatorname{alb}_{S}$ has connected fibers and its image is a smooth curve $B$ of genus $g(B)=q(S)>1$. Moreover, we have $q(X)=q(S)$ by Lemma 5.1. We distinguish two subcases: $q(S)=1$ and $q(S)>1$. If $q(S)=1$, then the image of $\mathrm{alb}_{X}$ is an elliptic curve and the same argument of the previous case applies. If $q(S)=g(B)>1$, then we get $V^{0}\left(\omega_{B}\right)=\operatorname{Pic}^{0}(B)$ and $\operatorname{Pic}^{0}(X) \cong \operatorname{Pic}^{0}(S) \cong \operatorname{Pic}^{0}(B)$. Hence, by Lemma 6.3, there are inclusions

$$
\operatorname{alb}_{S}^{*} \operatorname{Pic}^{0}(B)=\operatorname{alb}_{S}^{*} V^{0}\left(\omega_{B}\right) \subset V^{1}\left(\omega_{S}\right) \subset \operatorname{Pic}^{0}(S)
$$

leading to $V^{1}\left(\omega_{S}\right)=\operatorname{Pic}^{0}(S)$. Moreover, a second application of Lemma 6.3 gives

$$
f^{*} V^{1}\left(\omega_{S}\right) \subset V^{2}\left(\omega_{X}\right) \subset \operatorname{Pic}^{0}(X),
$$

showing that $V^{2}\left(\omega_{X}\right)=\operatorname{Pic}^{0}(X)$. Finally, we also have $V^{1}\left(\omega_{X}\right)=\operatorname{Pic}^{0}(X)$ as $\chi\left(\omega_{X}\right)=0$. 
Proposition 6.7. Let $X$ be a smooth projective threefold such that $\kappa(X)=1$, $\chi\left(\omega_{X}\right)=0$ and $V^{0}\left(\omega_{X}\right) \subsetneq \operatorname{Pic}^{0}(X)$.

(i) Assume $\operatorname{dim} \operatorname{alb}_{X}(X)=2$. If $q(X)=2$, then we have (i) $\operatorname{dim} V^{2}\left(\omega_{X}\right)=0$, (ii) $\operatorname{dim} V^{1}\left(\omega_{X}\right)=1$ if and only if $\operatorname{dim} V^{0}\left(\omega_{X}\right)=1$ and (iii) $\operatorname{dim} V^{1}\left(\omega_{X}\right)=0$ if and only if $\operatorname{dim} V^{0}\left(\omega_{X}\right) \leq 0$. If $q(X) \geq 3$, then we have $\operatorname{dim} V^{1}\left(\omega_{X}\right)=$ $\operatorname{dim} V^{2}\left(\omega_{X}\right)=q(X)-1$.

(ii) Assume $\operatorname{dim} \operatorname{alb}_{X}(X)=1$. If $q(X)=1$, then we have $\operatorname{dim} V^{1}\left(\omega_{X}\right) \leq 0$ and $\operatorname{dim} V^{2}\left(\omega_{X}\right)=0$. If $q(X) \geq 2$, then we obtain $V^{1}\left(\omega_{X}\right)=V^{2}\left(\omega_{X}\right)=\operatorname{Pic}^{0}(X)$.

Proof. We start with the case $\operatorname{dim}_{\operatorname{alb}_{X}}(X)=2$. Let $f: X \rightarrow C$ be a nonsingular representative of the litaka fibration of $X$ where $C$ is a smooth curve.

If $g(C) \geq 2$, then by Lemma 5.1 we have $q(X)=g(C)+1 \geq 3$, and by Lemma 6.3, we obtain a series of inclusions $f^{*} \operatorname{Pic}^{0}(C)=f^{*} V^{0}\left(\omega_{C}\right) \subset V^{2}\left(\omega_{X}\right) \subset \operatorname{Pic}^{0}(X)$. We conclude that

$$
\operatorname{dim} V^{2}\left(\omega_{X}\right)=q(X)-1
$$

since $V^{2}\left(\omega_{X}\right) \subsetneq \operatorname{Pic}^{0}(X)$ by (5). Therefore, we see that $V^{1}\left(\omega_{X}\right) \subsetneq \operatorname{Pic}^{0}(X)$ as $\chi\left(\omega_{X}\right)=0$ and $V^{0}\left(\omega_{X}\right) \subsetneq \operatorname{Pic}^{0}(X)$. Finally, thanks to the inclusion $V^{1}\left(\omega_{X}\right) \supset$ $V^{2}\left(\omega_{X}\right)$ of (6), we obtain $\operatorname{dim} V^{1}\left(\omega_{X}\right)=q(X)-1$.

If $g(C) \leq 1$, then $q(X)=2$ and $a:=\operatorname{alb}_{X}$ is surjective. Let $b: X^{\prime} \rightarrow Z^{\prime}$ be a nonsingular representative of the Stein factorization of $a$. Then, as we have seen in the proof of Proposition 6.5, $Z^{\prime}$ is birational to an abelian surface, and therefore, $\operatorname{dim} V^{2}\left(\omega_{X}\right)=0$. Since $\mathscr{O}_{X} \in V^{1}\left(\omega_{X}\right)$, we obtain the statements (ii) and (iii) of part (i).

We now study the case $\operatorname{dim} \operatorname{alb}_{X}(X)=1$. If $g(C) \geq 2$, then $q(X)=g(C)$ and $f^{*} \operatorname{Pic}^{0}(C)=\operatorname{Pic}^{0}(X)$. Therefore, by Lemma 6.3, we get $V^{2}\left(\omega_{X}\right)=\operatorname{Pic}^{0}(X)$, and hence, we have $V^{1}\left(\omega_{X}\right)=\operatorname{Pic}^{0}(X)$. On the other hand, if $g(C) \leq 1$, then $q(X)=1$ and $\operatorname{alb}_{X}: X \rightarrow \operatorname{Alb}(X)$ is an algebraic fiber space onto an elliptic curve. We conclude then as in the proof of Proposition 6.6.

Proposition 6.8. Let $X$ be a smooth projective threefold such that $\kappa(X)=0$ and $\chi\left(\omega_{X}\right)=0$. If $\operatorname{dim} \operatorname{alb}_{X}(X)=2$, then we have $\operatorname{dim} V^{1}\left(\omega_{X}\right)=\operatorname{dim} V^{2}\left(\omega_{X}\right)=0$. On the other hand, if $\operatorname{dim}_{a^{\prime}}(X)=1$, then we have $\operatorname{dim} V^{1}\left(\omega_{X}\right) \leq 0$ and $\operatorname{dim} V^{2}\left(\omega_{X}\right)=0$.

Proof. We recall that, by [Chen and Hacon 2002, Lemma 3.1], $V^{0}\left(\omega_{X}\right)$ consists of at most one point. We start with the case $\operatorname{dim} \operatorname{alb}_{X}(X)=2$. By [Kawamata 1981, Theorem 1], $\mathrm{alb}_{X}$ is surjective and has connected fibers. Therefore, we have $q(X)=h^{2}\left(X, \omega_{X}\right)=2$ and hence $\mathscr{O}_{X} \in V^{1}\left(\omega_{X}\right)$ since $\chi\left(\omega_{X}\right)=0$. We set $a:=\operatorname{alb}_{X}$, and we note that, by [Hacon 2004, Corollary 4.2], $a_{*} \omega_{X}$ is a GV-sheaf, i.e.,

$$
\operatorname{codim} V^{1}\left(a_{*} \omega_{X}\right) \geq 1 \quad \text { and } \operatorname{codim} V^{2}\left(a_{*} \omega_{X}\right) \geq 2 \text {. }
$$


By using that $R^{1} a_{*} \omega_{X} \cong \mathcal{O}_{\mathrm{Alb}(X)}$ and $R^{2} a_{*} \omega_{X}=0$ [Kollár 1986a, Proposition 7.6 and Theorem 2.1] and by using [Kollár 1986b, Theorem 3.1], we get isomorphisms

$$
H^{1}\left(X, \omega_{X} \otimes a^{*} L\right) \cong H^{1}\left(\operatorname{Alb}(X), a_{*} \omega_{X} \otimes L\right) \oplus H^{0}(\operatorname{Alb}(X), L)
$$

for any $L \in \operatorname{Pic}^{0}(\operatorname{Alb}(X)) \cong \operatorname{Pic}^{0}(X)$. Therefore, we have

$$
\operatorname{codim} V^{1}\left(\omega_{X}\right) \geq 1 \text { and } \operatorname{codim} V^{2}\left(\omega_{X}\right) \geq 2,
$$

and consequently, the hypothesis $\chi\left(\omega_{X}\right)=0$ implies $\operatorname{dim} V^{1}\left(\omega_{X}\right)=0$.

If $\operatorname{dim} \operatorname{alb}_{X}(X)=1$, then as in the previous case we have $\operatorname{dim} V^{2}\left(\omega_{X}\right)=0$. Therefore, $V^{1}\left(\omega_{X}\right)$ is either empty or of dimension zero since $\chi\left(\omega_{X}\right)=0$.

Proposition 6.9. Let $X$ be a smooth projective threefold such that $\kappa(X)=-\infty$ and $\chi\left(\omega_{X}\right)=0$.

(i) Suppose dimalb $\operatorname{alb}_{X}(X)=2$. If $q(X)=2$, then $V^{1}\left(\omega_{X}\right)=V^{2}\left(\omega_{X}\right)=\left\{\mathcal{O}_{X}\right\}$. If $q(X)>2$, then we obtain $\operatorname{dim} V^{1}\left(\omega_{X}\right)=\operatorname{dim} V^{2}\left(\omega_{X}\right)=q(X)-1$.

(ii) Suppose $\operatorname{dim} \operatorname{alb}_{X}(X)=1$. If $q(X)=1$, then we have $\operatorname{dim} V^{1}\left(\omega_{X}\right) \leq 0$ and $\operatorname{dim} V^{2}\left(\omega_{X}\right)=0$. If $q(X)>1$, then we obtain $V^{1}\left(\omega_{X}\right)=V^{2}\left(\omega_{X}\right)=\overline{\operatorname{Pic}}^{0}(X)$.

Proof. We start with the case $\operatorname{dim}_{\operatorname{alb}_{X}}(X)=2$. Let $a: X \rightarrow S \subset \operatorname{Alb}(X)$ be the Albanese map of $X$ and $b: X \rightarrow S^{\prime}$ be the Stein factorization of $a$, and let $c: X^{\prime} \rightarrow S^{\prime \prime}$ be a nonsingular representative of $b$. We can easily check that $q\left(X^{\prime}\right)=q\left(S^{\prime \prime}\right)$ and $\operatorname{dim} \operatorname{alb}_{S}(S)=2$ and hence that $\kappa\left(S^{\prime \prime}\right) \geq 0$. Furthermore, we have $c_{*} \omega_{X^{\prime}}=0$. To see this, we point out that by [Pareschi and Popa 2011, Theorem 5.8] $c_{*} \omega_{X^{\prime}}$ is a GV-sheaf on $S^{\prime \prime}$ and moreover that, by Lemma $6.4, V^{0}\left(c_{*} \omega_{X^{\prime}}\right)=V^{0}\left(\omega_{X^{\prime}}\right)=V^{0}\left(\omega_{X}\right)=\varnothing$. This immediately implies $c_{*} \omega_{X^{\prime}}=0$ as a GV-sheaf $\mathscr{F}$ is nonzero if and only if $V^{0}(\mathscr{F}) \neq \varnothing$. We distinguish now three cases according to the values of $\kappa\left(S^{\prime \prime}\right)$.

If $\kappa\left(S^{\prime \prime}\right)=0$, then $S^{\prime \prime}$ is birational to an abelian surface. This forces $q(X)=$ $q\left(X^{\prime}\right)=q\left(S^{\prime \prime}\right)=2$ and $c^{*} \operatorname{Pic}^{0}\left(S^{\prime \prime}\right)=\operatorname{Pic}^{0}\left(X^{\prime}\right)$. By [Kollár 1986b, Theorem 3.1; 1986a, Theorem 2.1 and Proposition 7.6], we obtain isomorphisms

$H^{2}\left(X^{\prime}, \omega_{X^{\prime}} \otimes c^{*} L\right) \cong H^{1}\left(S^{\prime \prime}, \omega_{S^{\prime \prime}} \otimes L\right) \quad$ and $\quad H^{1}\left(X^{\prime}, \omega_{X^{\prime}} \otimes c^{*} L\right) \cong H^{0}\left(S^{\prime \prime}, \omega_{S^{\prime \prime}} \otimes L\right)$ for any $L \in \operatorname{Pic}^{0}\left(S^{\prime \prime}\right)$. Therefore, we have $V^{2}\left(\omega_{X}\right) \cong V^{2}\left(\omega_{X^{\prime}}\right)=c^{*} V^{1}\left(\omega_{S^{\prime \prime}}\right)=\left\{\mathcal{O}_{X^{\prime}}\right\}$ and $V^{1}\left(\omega_{X}\right) \cong V^{1}\left(\omega_{X^{\prime}}\right)=c^{*} V^{0}\left(\omega_{S^{\prime \prime}}\right)=\left\{\mathcal{O}_{X^{\prime}}\right\}$.

If $\kappa\left(S^{\prime \prime}\right)=1$, then $S^{\prime \prime}$ is birational to an elliptic surface of maximal Albanese dimension fibered onto a curve of genus $g(C) \geq 2$. Thus, $X$ is fibered onto $C$ as well and $q\left(X^{\prime}\right)=q\left(S^{\prime \prime}\right)=g(C)+1$. By Lemma 6.3 and (5), we deduce $\operatorname{dim} V^{2}\left(\omega_{X^{\prime}}\right)=g(C)=q\left(X^{\prime}\right)-1$, and therefore, we get $\operatorname{dim} V^{1}\left(\omega_{X^{\prime}}\right)=q\left(X^{\prime}\right)-1$ as $\chi\left(\omega_{X^{\prime}}\right)=0$ and $V^{0}\left(\omega_{X^{\prime}}\right)=\varnothing$.

If $\kappa\left(S^{\prime \prime}\right)=2$, then by Castelnuovo's theorem we have $\chi\left(\omega_{S^{\prime \prime}}\right)>0$, which immediately yields $V^{0}\left(\omega_{S^{\prime \prime}}\right)=\operatorname{Pic}^{0}\left(S^{\prime \prime}\right)$. By using Lemma 6.3 , we see that $\operatorname{dim} V^{0}\left(\omega_{X^{\prime}}\right)>0$. This contradicts Lemma 6.4, and hence, this case does not occur. 
We now suppose $\operatorname{dim} \operatorname{alb}_{X}(X)=1$. Let $a: X \rightarrow C \subset \operatorname{Alb}(X)$ be the Albanese map of $X$ where $C:=\operatorname{Im} a$. Then $a$ has connected fibers and $q(X)=g(C)$ by [Ueno 1973, Lemma 2.11]. As in the previous case, we note that $a_{*} \omega_{X}=0$. Moreover, by [Kollár 1986b, Theorem 3.1; 1986a, Proposition 7.6], we obtain isomorphisms

$$
\begin{aligned}
& H^{1}\left(X, \omega_{X} \otimes a^{*} L\right) \cong H^{0}\left(C, R^{1} a_{*} \omega_{X} \otimes L\right), \\
& H^{2}\left(X, \omega_{X} \otimes a^{*} L\right) \cong H^{1}\left(C, R^{1} a_{*} \omega_{X} \otimes L\right) \oplus H^{0}\left(C, \omega_{C} \otimes L\right)
\end{aligned}
$$

for any $L \in \operatorname{Pic}^{0}(C)$. At this point, we distinguish two cases: $g(C)=1$ and $g(C)>1$. If $g(C)=q(X)>1$, then we have $V^{0}\left(\omega_{C}\right)=\operatorname{Pic}^{0}(C)$, and by Lemma 6.3, we get $V^{2}\left(\omega_{X}\right)=V^{1}\left(\omega_{X}\right)=\operatorname{Pic}^{0}(X)$. On the other hand, if $g(C)=q(X)=1$, then by [Hacon 2004, Corollary 4.2] $R^{1} a_{*} \omega_{X}$ is a GV-sheaf on $C=\operatorname{Alb}(X)$. Hence, we obtain $\operatorname{dim} V^{2}\left(\omega_{X}\right)=0$, and consequently, we see that $\operatorname{dim} V^{1}\left(\omega_{X}\right) \leq 0$ since $\chi\left(\omega_{X}\right)=0$ and $V^{0}\left(\omega_{X}\right)=\varnothing$.

Remark 6.10. In the case $q(X)=1$, the previous propositions yield the following statement: for each $k, \operatorname{dim} V^{k}\left(\omega_{X}\right)=1$ if and only if $\operatorname{dim} V^{k}\left(\omega_{Y}\right)=1$. In general, we have not been able to show that, if a locus $V^{k}\left(\omega_{X}\right)$ is empty or of dimension zero, then the corresponding locus $V^{k}\left(\omega_{Y}\right)$ is empty or of dimension zero, respectively. This ambiguity is mainly caused by the possible presence of nontrivial automorphisms.

An application of a sheafified version of the derivative complex [Ein and Lazarsfeld 1997, Theorem 3; Lazarsfeld and Popa 2010] can be shown to yield Conjecture 1.2 for threefolds having $q(X)=2$ [Lombardi 2013, Proposition 5.2.15].

\section{Applications}

In this final section, we prove Corollaries 1.7, 1.8 and 1.9. Moreover, we present a further result regarding the invariance of the Euler characteristic of powers of the canonical bundle for derived equivalent smooth minimal varieties of maximal Albanese dimension.

\section{A. Holomorphic Euler characteristic and Hodge numbers.}

Proof of Corollary 1.7. Let $d:=\operatorname{dim} X=\operatorname{dim} Y$. We begin with the case $\operatorname{dim} \operatorname{alb}_{X}(X)=d$. By Theorem 1.6, $Y$ is of maximal Albanese dimension, and by (5), we get inequalities

$$
\operatorname{codim} V^{1}\left(\omega_{X}\right) \geq 1 \quad \text { and } \operatorname{codim} V^{1}\left(\omega_{Y}\right) \geq 1 .
$$

We distinguish two cases: $V^{0}\left(\omega_{X}\right) \subsetneq \operatorname{Pic}^{0}(X)$ and $V^{0}\left(\omega_{X}\right)=\operatorname{Pic}^{0}(X)$. If $V^{0}\left(\omega_{X}\right) \subsetneq$ $\operatorname{Pic}^{0}(X)$, then we also have $V^{0}\left(\omega_{Y}\right) \subsetneq \operatorname{Pic}^{0}(Y)$ by Proposition 3.1. Moreover, there are inclusions $\operatorname{Pic}^{0}(X) \supsetneq V^{0}\left(\omega_{X}\right) \supset V^{1}\left(\omega_{X}\right) \supset \cdots \supset V^{d}\left(\omega_{X}\right)=\left\{\mathcal{O}_{X}\right\}$ and similarly for the loci $V^{k}\left(\omega_{Y}\right)$ (see (6)). Therefore, if $L \notin V^{0}\left(\omega_{X}\right)$ and $M \notin V^{0}\left(\omega_{Y}\right)$, then 
$h^{k}\left(X, \omega_{X} \otimes L\right)=h^{k}\left(Y, \omega_{Y} \otimes M\right)=0$ for all $k \geq 0$. Since the holomorphic Euler characteristic is invariant under deformation, we finally obtain

$$
\chi\left(\omega_{X}\right)=\chi\left(\omega_{X} \otimes L\right)=0=\chi\left(\omega_{Y} \otimes M\right)=\chi\left(\omega_{Y}\right) .
$$

On the other hand, if $V^{0}\left(\omega_{X}\right)=\operatorname{Pic}^{0}(X)$, then, by Proposition 3.1, $F\left(1, \operatorname{Pic}^{0}(X)\right)=$ $\left(1, \operatorname{Pic}^{0}(Y)\right)$, and thus,

there exists $L_{0} \in V^{0}\left(\omega_{X}\right) \backslash\left(\bigcup_{k=1}^{d} V^{k}\left(\omega_{X}\right)\right)$

$$
\text { such that } F\left(1, L_{0}\right)=\left(1, M_{0}\right) \text { with } M_{0} \in V^{0}\left(\omega_{Y}\right) \backslash\left(\bigcup_{k=1}^{d} V^{k}\left(\omega_{Y}\right)\right) \text {. }
$$

Hence, by using Corollary 2.2 with $m=k=0$, we have

$\chi\left(\omega_{X}\right)=\chi\left(\omega_{X} \otimes L_{0}\right)=h^{0}\left(X, \omega_{X} \otimes L_{0}\right)=h^{0}\left(Y, \omega_{Y} \otimes M_{0}\right)=\chi\left(\omega_{Y} \otimes M_{0}\right)=\chi\left(\omega_{Y}\right)$.

We suppose now $\operatorname{dim} \operatorname{alb}_{X}(X)=d-1$ and $\kappa(X) \geq 0$. By Theorem 1.6, we have $\operatorname{dim} \operatorname{alb}_{Y}(Y)=d-1$, and therefore, there are inclusions $V^{1}\left(\omega_{X}\right) \supset V^{2}\left(\omega_{X}\right) \supset \cdots \supset$ $V^{d}\left(\omega_{X}\right)$ and $V^{1}\left(\omega_{Y}\right) \supset V^{2}\left(\omega_{Y}\right) \supset \cdots \supset V^{d}\left(\omega_{Y}\right)$. We distinguish four cases.

The first case is when $V^{0}\left(\omega_{X}\right)=V^{1}\left(\omega_{X}\right)=\operatorname{Pic}^{0}(X)$. By Proposition 3.1 and Corollary 3.4, it turns out that $V^{0}\left(\omega_{Y}\right)=V^{1}\left(\omega_{Y}\right)=\operatorname{Pic}^{0}(Y)$ as well. We claim that there exists $0_{X} \neq L_{1} \in V^{0}\left(\omega_{X}\right) \backslash V^{2}\left(\omega_{X}\right)$

$$
\text { such that } F\left(1, L_{1}\right)=\left(1, M_{1}\right) \text { with } O_{Y} \neq M_{1} \in V^{0}\left(\omega_{Y}\right) \backslash V^{2}\left(\omega_{Y}\right) \text {. }
$$

In fact, the Rouquier isomorphism maps $F\left(1, \operatorname{Pic}^{0}(X)\right)=\left(1, \operatorname{Pic}^{0}(Y)\right)$ by Remark 3.6, and therefore, it is enough to choose the image under $F^{-1}$ of a generic element $(1, M)$ with $M \notin V^{2}\left(\omega_{Y}\right)$. By using Corollary 2.2 twice, first with $k=0$ and then with $k=1$, we obtain

$$
\begin{aligned}
\chi\left(\omega_{X}\right)=\chi\left(\omega_{X} \otimes\right. & \left.L_{1}\right)=h^{0}\left(X, \omega_{X} \otimes L_{1}\right)-h^{1}\left(X, \omega_{X} \otimes L_{1}\right) \\
& =h^{0}\left(Y, \omega_{Y} \otimes M_{1}\right)-h^{1}\left(Y, \omega_{Y} \otimes M_{1}\right)=\chi\left(\omega_{Y} \otimes M_{1}\right)=\chi\left(\omega_{Y}\right) .
\end{aligned}
$$

The second case is when $V^{0}\left(\omega_{X}\right)=\operatorname{Pic}^{0}(X)$ and $V^{1}\left(\omega_{X}\right) \subsetneq \operatorname{Pic}^{0}(X)$. By Proposition 3.1 and Corollary 3.4, $V^{0}\left(\omega_{Y}\right)=\operatorname{Pic}^{0}(Y)$ and $V^{1}\left(\omega_{Y}\right) \subsetneq \operatorname{Pic}^{0}(Y)$. As before, $F\left(1, \operatorname{Pic}^{0}(X)\right)=\left(1, \operatorname{Pic}^{0}(Y)\right)$, and hence, we can pick an element $\mathrm{O}_{X} \neq L_{2} \in V^{0}\left(\omega_{X}\right) \backslash V^{1}\left(\omega_{X}\right)$

$$
\text { such that } F\left(1, L_{2}\right)=\left(1, M_{2}\right) \text { with } \mathscr{O}_{Y} \neq M_{2} \in V^{0}\left(\omega_{Y}\right) \backslash V^{1}\left(\omega_{Y}\right) \text {. }
$$

Hence, equalities $\chi\left(\omega_{X}\right)=\chi\left(\omega_{X} \otimes L_{2}\right)=h^{0}\left(X, \omega_{X} \otimes M_{2}\right)=h^{0}\left(Y, \omega_{Y} \otimes M_{2}\right)=$ $\chi\left(\omega_{Y} \otimes M_{2}\right)=\chi\left(\omega_{Y}\right)$ hold.

The third case is when $V^{0}\left(\omega_{X}\right) \subsetneq \operatorname{Pic}^{0}(X)$ and $V^{1}\left(\omega_{X}\right)=\operatorname{Pic}^{0}(X)$. By using Proposition 3.1 and Corollary 3.4, it is easy to see that $V^{0}\left(\omega_{Y}\right) \subsetneq \operatorname{Pic}^{0}(Y)$ and 
$V^{1}\left(\omega_{Y}\right)=\operatorname{Pic}^{0}(Y)$. Moreover, Remark 3.6 yields $F\left(1, \operatorname{Pic}^{0}(X)\right)=\left(1, \operatorname{Pic}^{0}(Y)\right)$. Therefore, similarly to the previous cases, there exists a pair $\left(L_{3}, M_{3}\right) \neq\left(\mathrm{O}_{X}, \mathrm{O}_{Y}\right)$ such that

$$
F\left(1, L_{3}\right)=\left(1, M_{3}\right) \text { with } L_{3} \notin V^{0}\left(\omega_{X}\right) \cup V^{2}\left(\omega_{X}\right) \text { and } M_{3} \notin V^{0}\left(\omega_{Y}\right) \cup V^{2}\left(\omega_{Y}\right) \text {, }
$$

and by Corollary 2.2, we have $\chi\left(\omega_{X}\right)=\chi\left(\omega_{X} \otimes L_{3}\right)=-h^{1}\left(X, \omega_{X} \otimes L_{3}\right)=$ $-h^{1}\left(Y, \omega_{Y} \otimes M_{3}\right)=\chi\left(\omega_{Y} \otimes M_{3}\right)=\chi\left(\omega_{Y}\right)$.

The last case is when both $V^{0}\left(\omega_{X}\right)$ and $V^{1}\left(\omega_{X}\right)$ are proper subvarieties of $\operatorname{Pic}^{0}(X)$. Then $V^{0}\left(\omega_{Y}\right)$ and $V^{1}\left(\omega_{Y}\right)$ are proper subvarieties as well, and hence, $\chi\left(\omega_{X}\right)=\chi\left(\omega_{Y}\right)=0$.

Proof of Corollary 1.8. By the derived invariance of Hochschild homologies $H H_{0}(X) \cong H H_{0}(Y)$ and $H H_{1}(X) \cong H H_{1}(Y)$, we have $h^{0}\left(X, \omega_{X}\right)=h^{0}\left(Y, \omega_{Y}\right)$ and $h^{1}\left(X, \omega_{X}\right)=h^{1}\left(Y, \omega_{Y}\right)$. Therefore, Corollary 1.7 implies $h^{0,2}(X)=h^{0,2}(Y)$ since $h^{3}\left(X, \omega_{X}\right)=q(X)=q(Y)=h^{3}\left(Y, \omega_{Y}\right)$ and $h^{4}\left(X, \omega_{X}\right)=1=h^{4}\left(Y \omega_{Y}\right)$.

For the second equality, we apply Corollary 2.2 with $(L, M)=\left(\mathrm{O}_{X}, \mathrm{O}_{Y}\right)$ and $k=2$ so that $h^{2}\left(X, \omega_{X}\right)+h^{1}\left(X, \Omega_{X}^{3}\right)+h^{0}\left(X, \Omega_{X}^{2}\right)=h^{2}\left(Y, \omega_{Y}\right)+h^{1}\left(Y, \Omega_{Y}^{3}\right)+h^{0}\left(Y, \Omega_{Y}^{2}\right)$. Therefore, we obtain $h^{1,3}(X)=h^{1,3}(Y)$ since Serre duality and the Hodge linearconjugate isomorphism yield equalities $h^{2}\left(X, \omega_{X}\right)=h^{0}\left(X, \Omega_{X}^{2}\right)$ and $h^{2}\left(Y, \omega_{Y}\right)=$ $h^{0}\left(Y, \Omega_{Y}^{2}\right)$.

By using a result in [Pareschi and Popa 2011], we can also derive a consequence about pluricanonical bundles.

Corollary 7.1. Let $X$ and $Y$ be smooth projective derived equivalent varieties with $X$ of maximal Albanese dimension and minimal. Then

$$
\chi\left(\omega_{X}^{\otimes m}\right)=\chi\left(\omega_{Y}^{\otimes m}\right) \text { for all } m \geq 2 .
$$

Proof. By [Pareschi and Popa 2011, Corollary 5.5], $\omega_{X}^{\otimes m}$ and $\omega_{Y}^{\otimes m}$ are GVsheaves on $X$ and $Y$, respectively, for any $m \geq 2 .^{1}$ In particular, this implies that $\operatorname{codim} V^{1}\left(\omega_{X}^{\otimes m}\right) \geq 1$ and $\operatorname{codim} V^{1}\left(\omega_{Y}^{\otimes m}\right) \geq 1$. At this point, we argue as in the first part of the proof of Corollary 1.7 after having noted the inclusions $V^{0}\left(\omega_{X}^{\otimes m}\right) \supset V^{1}\left(\omega_{X}^{\otimes m}\right)$ and $V^{0}\left(\omega_{Y}^{\otimes m}\right) \supset V^{1}\left(\omega_{Y}^{\otimes m}\right)$ [Pareschi and Popa 2011, Proposition 3.14].

7B. Fibrations. In this subsection, we study the behavior of particular types of fibrations under derived equivalence. We begin by recalling some terminology from [Catanese 1991; Lazarsfeld and Popa 2010].

A smooth projective variety $X$ is of Albanese general type if it is of maximal Albanese dimension and has nonsurjective Albanese map. An irregular fibration or a higher irrational pencil is a surjective morphisms with connected fibers $f: X \rightarrow Z$

${ }^{1}$ The minimality condition is necessary; see [Pareschi and Popa 2011, Example 5.6]. 
onto a normal variety $Z$ with $0<\operatorname{dim} Z<\operatorname{dim} X$ and such that any smooth model of $Z$ is of maximal Albanese dimension or Albanese general type, respectively.

Popa [2013, Corollary 3.4] observes that a consequence of Conjecture 1.3 is that, if $X$ admits a fibration onto a variety having nonsurjective Albanese map, then any Fourier-Mukai partner of $X$ admits an irregular fibration. With Theorem 1.4 at hand, we can verify this statement under an additional hypothesis on $X$.

Proposition 7.2. Let $X$ and $Y$ be smooth projective derived equivalent varieties with $\operatorname{dim} \operatorname{alb}_{X}(X) \geq \operatorname{dim} X-1$. If $X$ admits a surjective morphism $f: X \rightarrow Z$ with connected fibers onto a normal variety $Z$ having nonsurjective Albanese map and such that $\operatorname{dim} X>\operatorname{dim} Z$, then $Y$ admits an irregular fibration.

Proof. Let $Z \stackrel{f^{\prime}}{\rightarrow} Z^{\prime} \rightarrow \operatorname{alb}_{Z}(Z)$ be the Stein factorization of $\operatorname{alb}_{Z}$. By taking a nonsingular representative of $f^{\prime}$, we can assume $Z^{\prime}$ smooth. We can easily check that $Z^{\prime}$ is of maximal Albanese dimension (so that $0_{Z^{\prime}} \in V^{0}\left(\omega_{Z^{\prime}}\right)$ ) and that $\mathrm{alb}_{Z^{\prime}}$ is not surjective. Hence, by [Ein and Lazarsfeld 1997, Proposition 2.2], there exists a positivedimensional irreducible component $V$ of $V^{0}\left(\omega_{Z^{\prime}}\right)$ passing through the origin. Moreover, by Lemma 6.3, we have $\left(f \circ f^{\prime}\right)^{*} V \subset V^{k}\left(\omega_{X}\right)_{0}$ where $k=\operatorname{dim} X-\operatorname{dim} Z^{\prime}$, and by (5), we get $\left(f \circ f^{\prime}\right)^{*} V \subset V^{k}\left(\omega_{X}\right)_{0} \subset V^{1}\left(\omega_{X}\right)_{0}$. Finally, by Theorem 1.4(iii), there exists a positive-dimensional irreducible component $V^{\prime} \subset V^{1}\left(\omega_{Y}\right)_{0}$ We conclude then by applying [Green and Lazarsfeld 1991, Theorem 0.1].

We point out that, thanks to Theorem 1.5 , we can remove the hypothesis "dim $\operatorname{alb}_{X}(X) \geq \operatorname{dim} X-1$ " from the above proposition in the case of threefolds. The following proposition, together with the subsequent remark, provides the proof of Corollary 1.9:

Proposition 7.3. Let $X$ and $Y$ be smooth projective derived equivalent threefolds. Fix $k$ to be either 1 or 2 . Then $X$ admits a higher irrational pencil $f: X \rightarrow Z$ with $0<\operatorname{dim} Z \leq k$ if and only if $Y$ admits a higher irrational pencil $g: Y \rightarrow W$ with $0<\operatorname{dim} W \leq k$.

Proof. We start with the case $k=1$, and therefore, we consider a higher irrational pencil $f: X \rightarrow Z$ onto a smooth curve $Z$ of genus $g(Z) \geq 2$. By Lemma 6.3, we have $f^{*} V^{0}\left(\omega_{Z}\right)=f^{*} \operatorname{Pic}^{0}(Z) \subset V^{2}\left(\omega_{X}\right)_{0}$, and by Theorem 1.5(i), there exists a component $T \subset V^{2}\left(\omega_{Y}\right)_{0}$ such that

$$
\operatorname{dim} T \geq q(Z) \geq 2
$$

Moreover, by [Green and Lazarsfeld 1991, Theorem 0.1] or by [Beauville 1992, Corollaire 2.3], there exists an irrational fibration $g: Y \rightarrow W$ onto a smooth curve $W$ such that $T \subset g^{*} \operatorname{Pic}^{0}(W)+\gamma$ for some $\gamma \in \operatorname{Pic}^{0}(Y)$. Therefore, we obtain the inequality

$$
q(W)=g(W) \geq \operatorname{dim} T \geq 2
$$


ensuring that $g$ is a higher irrational pencil.

We suppose now $k=2$, and we consider a higher irrational pencil $f: X \rightarrow Z$ onto a surface. It is a general fact that, by possibly replacing $Z$ with a lower-dimensional variety, one can furthermore assume $\chi\left(\omega_{Z^{\prime}}\right)>0$ for any smooth model $Z^{\prime}$ of $Z$ (see [Pareschi and Popa 2009, p. 271]). If $\operatorname{dim} Z=1$, then we apply the argument of the previous case. On the other hand, if $\operatorname{dim} Z=2$ then by Lemma 6.3 we get

$$
f^{*} V^{0}\left(\omega_{Z}\right)=f^{*} \operatorname{Pic}^{0}(Z) \subset V^{1}\left(\omega_{X}\right)_{0} .
$$

Moreover, by Theorem 1.5, there exists a component $T \subset V^{1}\left(\omega_{Y}\right)_{0}$ such that $\operatorname{dim} T \geq q\left(Z^{\prime}\right) \geq 3$, and by [Green and Lazarsfeld 1991, Theorem 0.1], there exists an irregular fibration $g: Y \rightarrow W$ such that $T \subset g^{*} \operatorname{Pic}^{0}(W)+\gamma$ for some $\gamma \in \operatorname{Pic}^{0}(Y)$. Therefore, $q(W) \geq \operatorname{dim} T \geq 3$ and $g$ is a higher irrational pencil.

Remark 7.4. We can slightly improve the statement of Proposition 7.3 in the case of fibrations onto curves. In fact, by going back to the proof of Proposition 7.3 in the case $k=1$, we see that from the inequalities (10) and (11) we obtain the inequality $q(W) \geq q(Z)$. Then the following holds. Fix an integer $g \geq 2$. The variety $X$ admits a higher irrational pencil $f: X \rightarrow C$ onto a curve of genus $g(C) \geq g$ if and only if $Y$ admits a higher irrational pencil $h: Y \rightarrow D$ onto a curve of genus $g(D) \geq g$.

\section{Acknowledgements}

I am deeply grateful to Mihnea Popa for his insights, hints and encouragement and to Christian Schnell for suggestions regarding the proof of Theorem 3.2. I also thank Chih-Chi Chou, Lawrence Ein, Víctor González-Alonso, Emanuele Macrì, Wenbo Niu and Tuan Pham for helpful conversations. This work got started and completed while I was a graduate student at the University of Illinois at Chicago.

\section{References}

[Arapura 1992] D. Arapura, "Higgs line bundles, Green-Lazarsfeld sets, and maps of Kähler manifolds to curves", Bull. Amer. Math. Soc. 26:2 (1992), 310-314. MR 92m:14010 Zbl 0759.14016

[Beauville 1992] A. Beauville, "Annulation du $H^{1}$ pour les fibrés en droites plats", pp. 1-15 in Complex algebraic varieties (Bayreuth, 1990), edited by K. Hulek et al., Lecture Notes in Math. 1507, Springer, Berlin, 1992. MR 94a:14048 Zbl 0792.14006

[Beauville 1996] A. Beauville, Complex algebraic surfaces, 2nd ed., London Mathematical Society Student Texts 34, Cambridge University Press, 1996. MR 97e:14045 Zbl 0849.14014

[Bridgeland and Maciocia 2001] T. Bridgeland and A. Maciocia, "Complex surfaces with equivalent derived categories", Math. Z. 236:4 (2001), 677-697. MR 2002a:14017 Zbl 1081.14023

[Brion 2010] M. Brion, "Some basic results on actions of nonaffine algebraic groups", pp. 1-20 in Symmetry and spaces, edited by H. E. A. Campbell et al., Progr. Math. 278, Birkhäuser, Boston, MA, 2010. MR 2011c:14128 Zbl 1217.14029 
[Catanese 1991] F. Catanese, "Moduli and classification of irregular Kaehler manifolds (and algebraic varieties) with Albanese general type fibrations", Invent. Math. 104:2 (1991), 263-289. MR 92f:32049 Zbl 0743.32025

[Chen and Hacon 2002] J. A. Chen and C. D. Hacon, "On algebraic fiber spaces over varieties of maximal Albanese dimension", Duke Math. J. 111:1 (2002), 159-175. MR 2002m:14007 Zbl 1055.14010

[Chen and Hacon 2004] J. A. Chen and C. D. Hacon, "On the irregularity of the image of the Iitaka fibration”, Comm. Algebra 32:1 (2004), 203-215. MR 2005c:14005 Zbl 1137.14008

[Căldăraru 2003] A. Căldăraru, “The Mukai pairing, I: The Hochschild structure”, preprint, 2003. arXiv math/0308079v2

[Ein and Lazarsfeld 1997] L. Ein and R. Lazarsfeld, "Singularities of theta divisors and the birational geometry of irregular varieties", J. Amer. Math. Soc. 10:1 (1997), 243-258. MR 97d:14063 Zbl 0901.14028

[Green and Lazarsfeld 1987] M. Green and R. Lazarsfeld, "Deformation theory, generic vanishing theorems, and some conjectures of Enriques, Catanese and Beauville", Invent. Math. 90:2 (1987), 389-407. MR 89b:32025 Zbl 0659.14007

[Green and Lazarsfeld 1991] M. Green and R. Lazarsfeld, "Higher obstructions to deforming cohomology groups of line bundles", J. Amer. Math. Soc. 4:1 (1991), 87-103. MR 92i:32021 Zbl 0735.14004

[Hacon 2004] C. D. Hacon, "A derived category approach to generic vanishing", J. Reine Angew. Math. 575 (2004), 173-187. MR 2005m:14026 Zbl 1137.14012

[Hacon and Pardini 2002] C. D. Hacon and R. Pardini, "On the birational geometry of varieties of maximal Albanese dimension”, J. Reine Angew. Math. 546 (2002), 177-199. MR 2003d:14016 Zbl 0993.14005

[Huybrechts 2006] D. Huybrechts, Fourier-Mukai transforms in algebraic geometry, Clarendon Press, Oxford, 2006. MR 2007f:14013 Zbl 1095.14002

[Kawamata 1981] Y. Kawamata, "Characterization of abelian varieties", Compositio Math. 43:2 (1981), 253-276. MR 83j:14029 Zbl 0471.14022

[Kawamata 2002] Y. Kawamata, "D-equivalence and $K$-equivalence”, J. Differential Geom. 61:1 (2002), 147-171. MR 2004m:14025 Zbl 1056.14021

[Kollár 1986a] J. Kollár, "Higher direct images of dualizing sheaves, I", Ann. of Math. (2) 123:1 (1986), 11-42. MR 87c:14038 Zbl 0598.14015

[Kollár 1986b] J. Kollár, "Higher direct images of dualizing sheaves, II", Ann. of Math. (2) 124:1 (1986), 171-202. MR 87k:14014 Zbl 0605.14014

[Lazarsfeld 2004] R. Lazarsfeld, Positivity in algebraic geometry, I, Ergebnisse der Mathematik und ihrer Grenzgebiete (3) 48, Springer, Berlin, 2004. MR 2005k:14001a Zbl 1093.14501

[Lazarsfeld and Popa 2010] R. Lazarsfeld and M. Popa, "Derivative complex, BGG correspondence, and numerical inequalities for compact Kähler manifolds", Invent. Math. 182:3 (2010), 605-633. MR 2011m:32024 Zbl 1209.32011

[Lombardi 2013] L. Lombardi, Derived equivalences of irregular varieties and constraints on Hodge numbers, Ph.D. thesis, University of Illinois at Chicago, 2013, http://www.math.uni-bonn.de/people/ lombardi/thesis.pdf.

[Matsumura 1963] H. Matsumura, "On algebraic groups of birational transformations", Atti Accad. Naz. Lincei Rend. Cl. Sci. Fis. Mat. Natur. (8) 34 (1963), 151-155. MR 28 \#3041 Zbl 0134.16601 
[Mori 1987] S. Mori, "Classification of higher-dimensional varieties", pp. 269-331 in Algebraic geometry, Bowdoin (Brunswick, ME, 1985), part 1, edited by S. J. Bloch, Proc. Sympos. Pure Math. 46, Amer. Math. Soc., Providence, RI, 1987. MR 89a:14040 Zbl 0656.14022

[Orlov 2003] D. O. Orlov, "Derived categories of coherent sheaves and equivalences between them", Uspekhi Mat. Nauk 58:3 (2003), 89-172. In Russian; translated in Russian Math. Surveys 58:3 (2003), 511-591. MR 2004g:14021 Zbl 1118.14021

[Pareschi and Popa 2009] G. Pareschi and M. Popa, "Strong generic vanishing and a higherdimensional Castelnuovo-de Franchis inequality", Duke Math. J. 150:2 (2009), 269-285. MR 2011b:14044 Zbl 1206.14067

[Pareschi and Popa 2011] G. Pareschi and M. Popa, "GV-sheaves, Fourier-Mukai transform, and generic vanishing”, Amer. J. Math. 133:1 (2011), 235-271. MR 2012e:14043 Zbl 1208.14015

[Pham 2011] T. D. Pham, On Picard varieties of surfaces with equivalent derived categories, $\mathrm{Ph} . \mathrm{D}$. thesis, University of Illinois at Chicago, 2011, http://search.proquest.com/docview/1286775745.

[Popa 2013] M. Popa, "Derived equivalence and non-vanishing loci", pp. 567-575 in A celebration of algebraic geometry (Cambridge, MA, 2011), edited by B. Hassett et al., Clay Math. Proc. 18, Amer. Math. Soc., Providence, RI, 2013. MR 3114957

[Popa and Schnell 2011] M. Popa and C. Schnell, "Derived invariance of the number of holomorphic 1-forms and vector fields”, Ann. Sci. Éc. Norm. Supér. (4) 44:3 (2011), 527-536. MR 2839458 Zbl 1221.14020

[Rosay 2009] F. Rosay, "Some remarks on the group of derived autoequivalences", preprint, 2009. arXiv 0907.3880

[Rouquier 2011] R. Rouquier, "Automorphismes, graduations et catégories triangulées", J. Inst. Math. Jussieu 10:3 (2011), 713-751. MR 2012h:16019 Zbl 1244.16009

[Swan 1996] R. G. Swan, "Hochschild cohomology of quasiprojective schemes", J. Pure Appl. Algebra 110:1 (1996), 57-80. MR 97j:19003 Zbl 0865.18010

[Toda 2006] Y. Toda, "Fourier-Mukai transforms and canonical divisors", Compos. Math. 142:4 (2006), 962-982. MR 2007d:14039 Zbl 1098.18005

[Ueno 1973] K. Ueno, “Classification of algebraic varieties, I", Compositio Math. 27 (1973), 277-342. MR 50 \#13030 Zbl 0284.14015

[Yekutieli 2003] A. Yekutieli, "The continuous Hochschild cochain complex of a scheme (survey)", pp. 227-233 in Topics in algebraic and noncommutative geometry (Luminy, France and Annapolis, MD, 2001), edited by C. G. Melles et al., Contemp. Math. 324, Amer. Math. Soc., Providence, RI, 2003. MR 1986127 Zbl 1064.16008

Communicated by Yujiro Kawamata

Received 2012-09-20 Revised 2013-06-03 Accepted 2013-09-28

lombardi@math.uni-bonn.de Mathematisches Institut, Universität Bonn, Endenicher Allee 60, 53115 Bonn, Germany 


\section{Algebra \& Number Theory}

msp.org/ant

\section{EDITORS}

MANAGING EDITOR

Bjorn Poonen

Massachusetts Institute of Technology

Cambridge, USA

\author{
EDITORIAL BOARD CHAIR \\ David Eisenbud \\ University of California \\ Berkeley, USA
}

\section{BOARD OF EDITORS}

Georgia Benkart

Dave Benson

Richard E. Borcherds

John H. Coates

J-L. Colliot-Thélène

Brian D. Conrad

Hélène Esnault

Hubert Flenner

Edward Frenkel

Andrew Granville

Joseph Gubeladze

Roger Heath-Brown

Ehud Hrushovski

Craig Huneke

Mikhail Kapranov

Yujiro Kawamata

János Kollár

Yuri Manin

Barry Mazur

Philippe Michel
University of Wisconsin, Madison, USA

University of Aberdeen, Scotland

University of California, Berkeley, USA

University of Cambridge, UK

CNRS, Université Paris-Sud, France

University of Michigan, USA

Freie Universität Berlin, Germany

Ruhr-Universität, Germany

University of California, Berkeley, USA

Université de Montréal, Canada

San Francisco State University, USA

Oxford University, UK

Hebrew University, Israel

University of Virginia, USA

Yale University, USA

University of Tokyo, Japan

Princeton University, USA

Northwestern University, USA

Harvard University, USA

École Polytechnique Fédérale de Lausanne
Susan Montgomery

Shigefumi Mori

Raman Parimala

Jonathan Pila

Victor Reiner

Karl Rubin

Peter Sarnak

Joseph H. Silverman

Michael Singer

Vasudevan Srinivas

J. Toby Stafford

Bernd Sturmfels

Richard Taylor

Ravi Vakil

Michel van den Bergh

Marie-France Vignéras

Kei-Ichi Watanabe

Efim Zelmanov

Shou-Wu Zhang
University of Southern California, USA

RIMS, Kyoto University, Japan

Emory University, USA

University of Oxford, UK

University of Minnesota, USA

University of California, Irvine, USA

Princeton University, USA

Brown University, USA

North Carolina State University, USA

Tata Inst. of Fund. Research, India

University of Michigan, USA

University of California, Berkeley, USA

Harvard University, USA

Stanford University, USA

Hasselt University, Belgium

Université Paris VII, France

Nihon University, Japan

University of California, San Diego, USA

Princeton University, USA

PRODUCTION

production@msp.org

Silvio Levy, Scientific Editor

See inside back cover or msp.org/ant for submission instructions.

The subscription price for 2014 is US $\$ 225 /$ year for the electronic version, and $\$ 400 /$ year $(+\$ 55$, if shipping outside the US) for print and electronic. Subscriptions, requests for back issues and changes of subscribers address should be sent to MSP.

Algebra \& Number Theory (ISSN 1944-7833 electronic, 1937-0652 printed) at Mathematical Sciences Publishers, 798 Evans Hall \#3840, c/o University of California, Berkeley, CA 94720-3840 is published continuously online. Periodical rate postage paid at Berkeley, CA 94704, and additional mailing offices.

ANT peer review and production are managed by EditFLOW ${ }^{\circledR}$ from Mathematical Sciences Publishers.

\section{PUBLISHED BY}

- mathematical sciences publishers

nonprofit scientific publishing

http://msp.org/

(C) 2014 Mathematical Sciences Publishers 


\section{Algebra \& Number Theory}

Volume $8 \quad$ No. $3 \quad 2014$

Derived invariants of irregular varieties and Hochschild homology

LUIGI LOMBARDI

Sato-Tate distributions of twists of $y^{2}=x^{5}-x$ and $y^{2}=x^{6}+1$

FRANCESC Fité and ANDREW V. SUTHERLAND

The algebraic dynamics of generic endomorphisms of $\mathbb{P}^{n}$

NAJMUDDIN FAKHRUDDIN

The tame-wild principle for discriminant relations for number fields

JOHN W. JONES and DAVID P. ROBERTS

Linear forms in logarithms and integral points on higher-dimensional varieties

AARON LEVIN

Lefschetz theorem for abelian fundamental group with modulus

689

MORITZ KERZ and ShuJi SAITO

Localization of spherical varieties

703

FRIEDRICH KNOP

Lefschetz operator and local Langlands modulo $\ell$ : the limit case

JEAN-FRANÇOIS DAT

Splitting tower and degree of tt-rings

PAUL BALMER 